\title{
An Analysis of the Guaranteed Withdrawal Benefits for Life Option
}

\author{
Premal Shah ${ }^{\dagger}$ and Dimitris Bertsimas ${ }^{\dagger \dagger}$
}

\begin{abstract}
In this paper, we present an analysis of the "Guaranteed Withdrawal Benefits" (GWB) for life option, a recent and popular product that many insurance companies are offering as a retirement planning solution. Under the GWB for life plan, the investor is promised increasing withdrawals during retirement for her lifetime, while still being invested in the markets. GWB for life thus transfers financial and longevity related risks from an individual investor to the insurance company underwriting the guarantee. We first analyze a continuous time version of this product in a Black Scholes economy with simplifying assumptions on population mortality and obtain an analytical solution for the product value. This analysis reveals the high sensitivity the product bears to several risk factors. We then price the GWB in a realistic setting using different asset pricing models, including those that allow the interest rates and the volatility of returns to be stochastic. Our analysis reveals that 1) GWB has insufficient price discrimination and is susceptible to adverse selection and 2) valuations can vary substantially depending on which class of models is used. We believe the ambiguity in value and the presence of significant risks, which can be challenging to hedge, should create concerns to the insurance companies offering the GWB, their investors and clients as well as the regulators.
\end{abstract}

\section{INTRODUCTION}

As a result of rising life expectancies as well as increases in lifestyle and health-care costs, retirement lifespans have become both longer and more expensive in the US. At the same time, with the social security system under considerable stress (see for example, Snow, [28]), the idea that individuals and households need to plan for their own retirement is gaining traction. Variable Annuity (VA) products are widely popular in the US as investment and tax-planning instruments. Companies offering VA products are now embedding them with options that will allow investors to also use them as a steady and assured source of income during retirement. More than $95 \%$ of VA products, whose total sales were over $\$ 180$ billion for the year 2007, now

Premal Shah is the corresponding author. email: premal@alum.mit.edu, Phone no.: +1-617-642-2232, Fax no.: +1-617258-9214.

${ }^{\dagger \dagger}$ Boeing Professor of Operations Research, Sloan School of Management, MIT, E40-147, Cambridge, MA 02139, USA; email: dbertsim@mit.edu. 
offer some sort of financial guarantees with them ${ }^{1}$. These guarantees generally offer the investor a form of downside protection against market risk and thus help her to secure her retirement nest-egg.

A recent innovation in this space was the "Guaranteed Minimum Withdrawal Benefit" (GMWB) offering. Under the GMWB plan, the investor's capital is invested through a VA fund in an asset-mix of her choice. The investor is guaranteed that upon maturity, which typically occurs at retirement, she will be able to take at least an $x \%$ of her initial investment every year for $N$ years, no matter how the underlying investment performs ${ }^{2}$. According to Milliman's third annual Guaranteed Living Benefits (GLB) survey of leading U.S. VA carriers, election rates of the GMWB scheme or its variants have increased steadily from $24 \%$ in 2004 to $29 \%$ in 2005, to $40 \%$ in 2006, and then to $43 \%$ during the first six months of 2007 and the GMWB family has now become the most popular of all VA options.

Insurance companies have now also started offering a lifetime benefit feature with GMWB, enabling the investor to simultaneously manage both financial as well as longevity related risks. GMWB with lifetime withdrawals is commonly known as "Guaranteed Lifetime Withdrawal Benefits" (GLWB) or "Guaranteed Withdrawal Benefits" (GWB) for life. GWB for life typically guarantees the investor withdrawal amounts that are indexed to her VA account's high watermark value (called the benefit base) for her entire life. A key advantage of the GWB feature over other VA based options available in the market is that in GWB, the underlying investment can continue to have market exposure even when the withdrawals start and thus has a greater growth opportunity. In contrast, with other widely marketed VA based options such as the Guaranteed Minimum Accumulation Benefit (GMAB) or the Guaranteed Minimum Income Benefit (GMIB)/ Guaranteed Annuity Option (GAO), the underlying investment is effectively annuitized or invested in fixed income instruments upon maturity. See Chu and Kwok [15], for more details about the GAO products.

Unlike exchange traded or over the counter options, the investor pays for an option elected with VAs in a piece-meal way over several years, typically as a fixed fraction of the underlying account value or the defined "protection" level. For the GWB for life, currently insurance companies are charging annual fees in the range of roughly 50 to 90 basis points. The fee is

1. Source: Milliman Research

2. Typically $x \cdot N=100$ so that the total guarantee is equal to the original investment. 
usually indexed to the benefit base that is used for calculating guaranteed withdrawal amounts ${ }^{3}$.

If fairly priced, the GWB for life option is an attractive retirement solution for investors as it allows them to manage the risks related to their own longevities, which cannot be mitigated at an individual level. Further, the ability to stay invested in the market while in retirement would allow investors to better cope with the inflation related risk, which becomes significant as the retirement lifespans get longer ${ }^{4}$.

For the long term sustainability of the GWB and related products, it is also important that the companies offering them remain profitable and viable. For example, GAO schemes, which are basically equity denominated interest rate options, were launched in the UK in a high interest rate environment, but as they drew closer to maturity interest rates plummeted while the stock markets soared, forcing at least one company to close its product to new buyers (Chu and Kwok, [15]). Also, if the GWB is significantly underpriced or raises the possibility of a debilitating loss for the underwriting company, then the related credit-worthiness issues should make potential clients skeptical of the GWB. This is because clients are likely to reap GWB for life's benefits only 20 to 30 years after enrolling for the product. As we show in this paper, such concerns will not be misplaced because the GWB for life feature entails considerable risk from a large number of factors that should pose serious challenges in its valuation and risk management.

\section{Related Work}

Brennan and Schwartz [12] and Boyle and Schwartz [11] were one of the first to extend option pricing methodology to insurance contracts where the time or frequency of payouts are linked to investor's death. Biffis [7], Chu and Kwok [15], Boyle and Hardy [10], Ballotta and Haberman [5], Pelsser [27] have analysed the GMIB/GAO type option feature offered in the VAs.

The GWB for life is a relatively recent addition to the market space, and is yet to be analyzed in detail in the literature. GMWB, which is similar to the GWB for life except that it guarantees withdrawals over only a fixed number of years, has been analyzed by Milevsky and Salisbury [25], Dai et al. [17], Chen et al. [14]. Milevsky and Salisbury [25] points out that GWMB is like a Quanto Asian Put Option in a Black-Scholes economy and find that it is possibly severely

3. VA based options in general differ in terms of promised withdrawal rates as well as features such as the frequency of "step-ups" of the benefit base and the indexing of the fees. http://www.annuityfyi.com provides a long but non-comprehensive list of leading insurance companies offering GWB for life like products.

4. Equity markets are known to be better hedges against inflation as compared to fixed income instruments like annuities over a long run, see, for example, Bodie [9]. 
underpriced. Dai, Kwok and Zong [17] analyze the same product from an investor's perspective and focus exclusively on deriving the optimal withdrawal policies, again in a Black-Scholes framework. Recently, Chen, Vetzal and Forsyth [14] studied the impact of optimal "withdrawal" strategies as well as jump risks in the context of GMWB. These papers do not consider the ratchet like step-up feature which is common in GWB for life guarantees. Another recent paper by Hoz, Kling and Rub, [20], presents a numerical analysis of the GWB for life with ratchet like features, also in a Black-Scholes framework. Valuations of the GWB/GMWB family have so far been based primarily on the basic Black-Scholes model.

\section{Approach, Findings and Contributions}

In this paper, we seek to value the GWB and investigate the associated risk-factors and their severity. We proceed by taking a typical GWB offering and pricing it under a series of valuation models. We start with a simple model to build intuition and then incrementally refine it to make it more realistic. This approach allows us to get an estimate of the magnitude of the impact the key risk factors can have.

- We first analyze a continuous time version of the GWB for life assuming retirement lifespans are exponentially distributed and Black-Scholes asset price dynamics. This model allows us to obtain an analytical expression for the value of GWB. While this analysis cannot be used as an absolute valuation tool for the GWB for life, it provides us valuable insights into the possible sources of risk. We find that:

- The GWB guarantees become more expensive for the company as the volatility of the underlying VA fund increases.

- The product value varies significantly depending on the investor age at inception creating an adverse selection bias risk.

- Since GWB for life has long durations that can easily extend over 30 - 40 years, interest rate related fluctuations may be important for pricing. Ballotta [4], Lee and Stock [22], Wang, Gerrard and Haberman [30] have pointed out the importance of considering interest rate risks in the VA products with embedded options, however this risk has not been analyzed in context of GWB like products which are primarily equity based. The GWB value has a convex relationship with interest rates making it susceptible to volatilities in interest rates as well.

- Cairns, Blake and Dowd [13], Biffis [6] and Milvesky, Promislow and Young [24] have argued that population mortality related risk may consist of a systemic component and 
hence may not be entirely diversifiable ${ }^{5}$. As one would expect, GWB for life also has a sizeable mortality related risk.

- We then take away the simplifying assumptions and price a typical GWB for life offering using a realistic distribution of retirement lifespans. Additionally, we augment the basic Black-Scholes model that has been traditionally used to price the GMWB family and other equity based VA products to incorporate the effect of stochastic interest-rates. We also investigate valuation under a stochastic volatility model, which implies fatter tails for the underlying VA fund returns. We ensure that the models are tuned so that they imply the same levels for the yield curve and option implied volatility of asset returns. We find that:

- Accounting for stochasticity in interest rates and return volatilities has a material downside effect on valuations.

- The value put to the GWB has a high dependence on the class of models used. As the GWB is not market traded, companies issuing GWB value them using their own proprietary models. Given its impressive sales, this "model risk" associated with the GWB implies that there is a high possibility of a significant mis-valuation of the insurance companies' liabilities due to model mis-specification.

\section{Paper Layout}

In Section 2 we formally describe the GWB for life option contract and its features. In Section 3, we present an analysis of the GWB under the simplifying assumptions mentioned earlier. This is followed by a numerical analysis in Section 4. In Section 5, we analyze GWB in a more realistic setting using three different arbitrage pricing models. Section 6 presents the implications of these models for GWB valuation. Section 7 summarizes the key findings.

\section{MODEL}

In this section, we formally describe the GWB for life product structure used in this paper.

- All guaranteed payments and fees are defined in terms of two state variables - one is simply the underlying VA account value and the other is referred to as the "benefit base". We use $B_{n}$ and $C_{n}$ to respectively denote the benefit base and the account value net of withdrawals, if any, at the $n^{\text {th }}$ anniversary.

5. For example, medical breakthroughs or natural or man-made calamities systemically impact a population's longevity. 
- At time 0 , a representative investor aged $A$ opens an account with the GWB for life feature with an initial investment $C_{0}$. This capital is invested in an asset mix of investor's choice through a VA fund. All dividends and distributions are assumed to be reinvested. For simplicity, we construct a reference index $S_{t}$ for the VA fund to tracks its total returns. The initial value of the benefit base is set as $B_{0}=C_{0}$.

- There is a minimum waiting period $W$ and a retirement age $A_{R}$ defined in the contract. The investor can start taking withdrawals from her account, starting from the $(T+1)^{s t}$ anniversary, where $T=\max \left(A_{R}-A, W\right)$. We assume $A, A_{R}, W$ to be all integers. For $n>T$, the investor is guaranteed to be able to take a withdrawal of $q \cdot B_{n-1}$ at the $n^{\text {th }}$ anniversary. The insurance company is responsible for covering any shortfall in case the account value, i.e., $C_{n}$ falls below the guaranteed withdrawal level, i.e., $q \cdot B_{n-1}$. This is the GWB guarantee.

- If the withdrawal taken at the $n^{t h}$ anniversary does not exceed the contractual guarantee (i.e., 0 for the first $T$ anniversaries and $q \cdot B_{n-1}$ thereafter), the benefit base $B_{n}$ is set to the higher of $B_{n-1}$ and the contract value after withdrawals, i.e., $C_{n}$. If the withdrawals exceed the contractual guarantee, then $B_{n}$ is set to the lower of $B_{n-1}$ and $C_{n}$. This is the step-up (also sometimes known as ratchet) feature.

- The investor is charged a fee $h \cdot B_{n-1}$ at the $n^{t h}$ anniversary for the GWB guarantee. We assume that the fees are charged separately to the investor rather than being deducted from the account ${ }^{6}$.

- Upon investor's death, the residual account value, if any, is returned to a beneficiary.

GWB valuation is clearly dependent on how the investor chooses to withdraw. In principle, the investor can strategize her withdrawals. Milevsky and Salisbury [25], Dai, Kwok and Zong [17] and Chen, Vetzal and Forsyth [14] investigate the implications of "optimal" dynamic withdrawals by investors and find them to increase the GMWB costs substantially. However, we believe that in practice, especially for the GWB for life type product, investors are unlikely to follow such optimal dynamic policies. This is because:

- "Optimal" withdrawal policies typically recommend the investor to withdraw her invest-

6. In practice, fees related to VA products are typically deducted from the investor's account every year and thus affect the account value. The assumption that the fees are charged separately allows us to isolate the cash inflows and outflows associated with the product. This helps us to compute the break even fees easily, which otherwise would need solving a fixed point problem. As the fee involved is small, typically few tens of basis points a year, we do not expect this approximation to alter our results in a significant way. 
ment out completely, when the net fair value of the protection guarantee to the investor turns negative. The papers cited above consider only the surrender charges that the insurance company levies on large withdrawals to evaluate the cost of this strategy for the investor. In practice, the investor will also incur high indirect costs in terms of taxes on the excess distributions and this is likely to make taking large strategic withdrawals unattractive.

- An individual investor is also more risk-averse, unable to hedge risks due to her own longevity and less equipped than large institutions like insurance companies to hedge financial risks.

Hence in our analysis, we assume that the investor withdraws the contractually guaranteed amount at each anniversary. This is the maximum withdrawal the investor can take without causing the step-ups to reverse. Let $R_{n+1}^{s}$ denote the return on the underlying VA fund for the period $(n, n+1]$. The dynamics of the account value $C_{n}$ and the benefit base $B_{n}$ for a steady contract specified rate of withdrawal are given by:

$$
\begin{aligned}
C_{n+1} & =\left(C_{n} \cdot R_{n+1}^{s}-q_{n+1} B_{n}\right)^{+} ; \\
B_{n+1} & =\max \left(B_{n}, C_{n+1}\right),
\end{aligned}
$$

where

$$
q_{n}=\left\{\begin{array}{l}
0, \text { if } n \leq T, \\
q, \text { if } n>T .
\end{array}\right.
$$

So far, we have not incorporated the mortality related randomness in our model. We assume that the mortality process is stationary and independent of the market dynamics, and the insurance company is risk-neutral with respect to it. We denote by $\mathbb{Q}$ the measure on the expanded sample space containing both the securities market and the investors' mortalities and which is obtained by combining the risk neutral pricing measure and the mortality laws. While we do not price the mortality risk into the GWB, we investigate the magnitude of the implied risk. This can be used to compute a premium or risk capital.

Before we proceed to a formal analysis, a simple backtesting experiment reveals some interesting facts about the GWB. Suppose an investor aged 60 had elected a typical GWB, with $q=6 \%$, $A_{R}=65$ years and $W=3$ years, sometime between 1950 and 1972 (assuming it was being offered then), and her VA account was invested in a steady mix of equities (more sepcifically, the the S\&P composite universe portfolio) and long term bonds by value, rebalanced monthly. In all possible scenarios, the guarantee provided by GWB would have been superfluous, even 
if the investor went on to live upto 95 years, i.e., the investor's account value would have been sufficient to finance the guaranteed level of withdrawals and the company would have had zero realized costs. Also, the more aggressive the asset mix chosen at inception and the longer the investor lived (upto 95 years), the higher would have been the revenues for the company. The picture and inferences will be very different if one considers a longer duration, in particular, the period from 1871 and 1972. Cash-flows involved can be computed using the monthly data for S\&P composite levels, dividends and long-term interest rates, as extrapolated by Shiller and available online from the website [1] for the period - January 1871 to December 2007. Although, the GWB still appears to be a profitable enterprise on the whole, there is a significant possibility of substantial losses. Moreover pofitability now decreases with the aggressiveness of the chosen asset mix and higher investor longevities no longer appear beneficial to the insurance company.

We now turn to a formal analysis of the GWB for life. From (1), it follows that the GWB has discrete cash-flows. Considerable analytical simplicity however is offered by considering a continuous time version of the product. In the next section, we consider this case and derive useful insights into the risk factors associated with the GWB for life.

\section{A First Analysis - Black Scholes Model with Continuous Step-ups and EXPONENTIAL MORTALITY}

For the analysis presented in this section, we make two key simplifying assumptions to achieve tractability:

- Step-ups as well as all cash-flows, i.e., withdrawals and fee payments happen continuously.

- Investor's death after retirement can be modeled as a Poisson arrival process with constant intensity $\lambda$. Moreover this process is independent of the market returns process.

We assume that $S_{t}$, the reference index for the chosen VA fund, follows a geometric Brownian motion with constant volatility $\sigma$ and the risk free rate $r$ is constant. Thus asset price dynamics are as in the Black-Scholes model [8]. The investor starts taking withdrawals from time $t>T \triangleq$ $\max \left(W, A_{R}-A\right)$ at the rate $q_{t} B_{t}$ where,

$$
q_{t}=\left\{\begin{array}{lll}
0, & \text { if } & t \leq T \\
q, & \text { if } & t>T .
\end{array}\right.
$$

The investor also (separately) pays fees to the company at a rate $h$ indexed to $B_{t}$. 
In this model, the only state variables that affect the GWB value are $B_{t}, C_{t}$ and the investor age or equivalently time $t$. The dynamics for $B_{t}$ and $C_{t}$ are given as:

$$
\begin{aligned}
& d C_{t}=\left\{\begin{array}{lll}
r C_{t} d t-q_{t} B_{t} d t+C_{t} \sigma d Z_{t}^{Q} & \ldots & C_{t} \geq 0 \\
0 & \ldots & C_{t}=0
\end{array},\right. \\
& d B_{t}=\mathbf{1}_{\left\{C_{t}=B_{t}\right\}}\left(d C_{t}\right)^{+},
\end{aligned}
$$

where $Z_{t}^{Q}$ is a $\mathbb{Q}$ Brownian motion. Now, let $L(C, B, t)$ denote the fair value of the protection offered to the investor through the GWB scheme at $t$ as a function of the state variables. This is simply that part of the withdrawals that are borne by the insurance company.

Let $G_{h}(C, B, t)$ denote the value of the revenue stream from the investor at time $t$. We also define a fee rate normalized revenue stream as $G(C, B, t) \triangleq \frac{G_{h}(C, B, t)}{h}$. Our goal is to find the functions $L(C, B, t)$ and $G(C, B, t)$.

We first note the following simple property that reduces the dimensionality of the problem. Proposition 1: $L(C, B, t)$ and $G(C, B, t)$ are homogeneous in $B, C$ and satisfy:

$$
\begin{aligned}
L(C, B, t) & =B \cdot L\left(\frac{C}{B}, 1, t\right) ; \\
G(C, B, t) & =B \cdot G\left(\frac{C}{B}, 1, t\right) .
\end{aligned}
$$

Proof: Let the tuple $\left(B_{t}, C_{t}\right)$ denote the investor's benefit base and account value at time $t$. For a given sample path $\omega$ of the evolution of the market and mortality factors, let $\left(B_{s}(\omega), C_{s}(\omega)\right)$ be the value of this tuple at some time $s \geq t$ along $\omega$. From the homogeneity of the system dynamics as given by (3) and (4), it follows that given some $y>0$, if the investor had started with a benefit base and account value combination $\left(y \cdot B_{t}, y \cdot C_{t}\right)$, then the sample path $\omega$ values of these quantities at some time $s \geq t$ would have been $\left(y \cdot B_{s}(\omega), y \cdot C_{s}(\omega)\right)$. Moreover, the cashflows that the company incurs, i.e., the shortfalls and revenues at any time $t$ are also homogeneous functions of the benefit base $B_{t}$ and account value $C_{t}$. From these, it follows that for any $y>0$,

$$
\begin{aligned}
L(y \cdot C, y \cdot B, t) & =y \cdot L(C, B, t) ; \\
G(y \cdot C, y \cdot B, t) & =y \cdot G(C, B, t) .
\end{aligned}
$$

The desired property follows immediately.

In light of Proposition 1, we define the functions $l(x, t)$ and $g(x, t)$ as follows

$$
\begin{aligned}
& l(x, t)=L(x, 1, t), \ldots, 0 \leq x \leq 1 ; \\
& g(x, t)=G(x, 1, t), \ldots, 0 \leq x \leq 1 .
\end{aligned}
$$


The quantity $x$ can be interpreted as the benefit base capitalization ratio $\frac{C}{B}$. The functions $l(x, t)$ and $g(x, t)$ are to be interpreted as the values of respectively the liabilities and revenue streams normalized by the benefit base, before a step-up operation is performed.

For $t \geq T$, it is convenient to find $L(C, B, t)$ and $G(C, B, t)$ (or equivalently $l(x, t)$ and $g(x, t)$ ) by solving a differential equation. Using these, $L(C, B, t)$ and $G(C, B, t)$ can then be determined for $t<T$ by taking a risk neutral expectation of their respective values at time $T$. We therefore break the pricing of GWB for life into two different phases:

1) Phase 1: When $t<T$. There are no withdrawals in this phase.

2) Phase 2: When $t \geq T$ and the investor has started taking withdrawals.

We now elaborate each of these in detail beginning with Phase 2.

\subsection{Value of Cashflows in Withdrawal Phase (Phase 2)}

Here, because of the assumption that the investor has an exponentially distributed residual life in retirement, $l(x, t)$ and $g(x, t)$ as defined in (6) become independent of time $t$ for $t \geq T$. We summarize this in the following proposition.

Proposition 2: For $t \geq T$,

$$
\begin{aligned}
& l(x, t)=l(x, T) ; \\
& g(x, t)=g(x, T) .
\end{aligned}
$$

Proof: Both the market returns process as well as the mortality process are memoryless for $t \geq T$. Moreover the dynamics of the state variables as given by (3) and (4) also do not depend on $t$ for $t \geq T$ as $q_{t}=q$ is a constant for this period. It then follows that for $t \geq T$, the system has no memory and hence

$$
\begin{aligned}
L(C, B, t) & =L(C, B, T) ; \\
G(C, B, t) & =G(C, B, T) .
\end{aligned}
$$

The result in (7) then follows immediately from (6).

For $t \geq T$, then $l(x, t)$ and $g(x, t)$ can be regarded as functions of just one variable. To ease notation, for this particular subsection where we are working under the case $t \geq T$, we shall denote them as simply $l(x)$ and $g(x)$ respectively.

Lemma 1: 1) The function $l(\cdot)$ satisfies the $2^{\text {nd }}$ order differential equation

$$
\frac{1}{2} \sigma^{2} x^{2} l^{\prime \prime}+(r x-q) l^{\prime}-(r+\lambda) l=0 ;
$$


and the boundary conditions

$$
\begin{aligned}
l(0) & =\frac{q}{r+\lambda}, \\
l^{\prime}(1) & =l(1) .
\end{aligned}
$$

2) The function $g(\cdot)$ satisfies the $2^{\text {nd }}$ order differential equation

$$
\frac{1}{2} \sigma^{2} x^{2} g^{\prime \prime}+(r x-q) g^{\prime}-(r+\lambda) g+1=0 ;
$$

and the boundary conditions

$$
\begin{aligned}
g(0) & =\frac{1}{r+\lambda}, \\
g^{\prime}(1) & =g(1) .
\end{aligned}
$$

Proof: We will prove the result only for the function $l(x)$, as the proof for $g(x)$ follows along identical lines. We know that in phase $2, L(C, B, t)=L(C, B, T)=B \cdot l\left(\frac{C}{B}\right)$. Let $A$ be the event that the investor dies in the interval $(t, t+d t]$. Thus $\mathbb{P}(A)=\lambda d t+o\left(d t^{2}\right)$. When $0<C<B$, $d B=0$. Then by Ito's Lemma,

$$
\begin{aligned}
d L & =\mathbb{P}(A) \cdot(0-L)+(1-\mathbb{P}(A)) \cdot\left(\frac{\partial L}{\partial C} d C+\frac{1}{2} \frac{\partial^{2} L}{\partial C^{2}}<d C \cdot d C>\right) \\
& =-\lambda L d t+\frac{\partial L}{\partial C}\left((r C-q B) d t+\sigma C d Z_{t}^{Q}\right)+\frac{\partial^{2} L}{\partial C^{2}} \sigma^{2} C^{2} d t .
\end{aligned}
$$

Here, $\left\langle d C \cdot d C>\right.$ is short-hand for $\mathbb{E}^{\mathbb{Q}}\left[d C^{2}\right]$. Under the risk-neutral measure, using the standard arguments for asset pricing (see Duffie [18]), we must have

$$
\begin{aligned}
\mathbb{E}^{\mathbb{Q}}[d L] & =r L d t-(q B d t-L)^{+}, \\
\text {i.e., }(r C-q B) \frac{\partial L}{\partial C}+\frac{1}{2} \sigma^{2} C^{2} \frac{\partial^{2} L}{\partial C^{2}}-\lambda L & =r L .
\end{aligned}
$$

Using the property that $L(C, B, t)=B \cdot l(x)$, with $x \triangleq \frac{C}{B}$, we get

$$
\begin{aligned}
(r x-q) l^{\prime}+\frac{1}{2} \sigma^{2} x^{2} l^{\prime \prime}-\lambda l & =r l, \\
\text { i.e., } \frac{1}{2} \sigma^{2} x^{2} l^{\prime \prime}+(r x-q) l^{\prime}-(r+\lambda) l & =0 .
\end{aligned}
$$

For the first boundary condition, we simply note that

$$
\begin{aligned}
l(0) & =L(0,1, T) \\
& =\mathbb{E}^{\mathbb{Q}}\left[\int_{0}^{\infty} \exp (-(r+\lambda) t) \cdot q d t\right]=\frac{q}{r+\lambda} .
\end{aligned}
$$

The second boundary condition is more technical and represents a "smooth pasting condition". We give a rough proof: 
We first assume that step-ups occur discretely at points spaced by a small interval $\Delta$. Suppose a step-up point just elapsed at time $t$. Let $B_{t}=B, C_{t+\Delta}=C$ and $L\left(C_{t+\Delta}, B_{t+\Delta}\right)=$ $L(C, \max (C, B)) \triangleq \tilde{L}_{B}(C)$. The function $\tilde{L}_{B}(\cdot)$ is assumed to be continuously differentiable in $C$. Then, it follows that,

$$
\text { if } C>B, \frac{d \tilde{L}_{B}(C)}{d C}=\frac{d L(C, C)}{d C}=l(1) .
$$

By continuity,

$$
\left.\frac{d \tilde{L}_{B}(C)}{d C}\right|_{C=B}=l(1)
$$

Now, since $B_{t}$ is continuous,

$$
\begin{aligned}
\lim _{\Delta \rightarrow 0} \tilde{L}_{B}(C) & =B l\left(\frac{C}{B}\right), \\
\text { hence, } \lim _{\Delta \rightarrow 0} \frac{d \tilde{L}_{B}(C)}{d C} & =l^{\prime}\left(\frac{C}{B}\right) .
\end{aligned}
$$

Putting (15) and (16) together, we get the boundary condition (10).

The differential equations in Lemma 8 have the following general solutions

$$
\begin{aligned}
l(x) & =C_{1} x^{-k} e^{-\frac{q}{\sigma^{2} x}} \operatorname{WhM}\left(k, m, \frac{2 q}{\sigma^{2} x}\right)+C_{2} x^{-k} e^{-\frac{q}{\sigma^{2} x}} \operatorname{WhW}\left(k, m, \frac{2 q}{\sigma^{2} x}\right), \\
g(x) & =D_{1} x^{-k} e^{-\frac{q}{\sigma^{2} x}} \operatorname{WhM}\left(k, m, \frac{2 q}{\sigma^{2} x}\right)+D_{2} x^{-k} e^{-\frac{q}{\sigma^{2} x}} \operatorname{WhW}\left(k, m, \frac{2 q}{\sigma^{2} x}\right)+\frac{1}{r+\lambda} ; \\
\text { where } k & =\frac{r}{\sigma^{2}}-1, \\
m & =\sqrt{\left(\frac{1}{2}+\frac{r}{\sigma^{2}}\right)^{2}+2 \frac{\lambda}{\sigma^{2}}} .
\end{aligned}
$$

The functions $\operatorname{WhW}(k, m, z)$ and $\operatorname{WhM}(k, m, z)$ are hypergeometric functions that solve the Whittaker Differential Equation (see, Abramowitz and Stegun, [2]).

$$
\frac{d^{2} u}{d z^{2}}+\frac{d u}{d z}+\left(\frac{k}{z}+\frac{\frac{1}{4}-m^{2}}{z^{2}}\right) u=0 .
$$

Some basic properties of these functions, as well as the $\operatorname{limits}_{z \rightarrow \infty} \operatorname{WhM}(k, m, z)$ and $\lim _{z \rightarrow \infty} \mathrm{WhW}(k, m, z)$ are provided in Appendix A. Using these properties, we can find the constants $C_{1}, C_{2}, D_{1}, D_{2}$ satisfying the boundary conditions given in Lemma 1 as

$$
\begin{aligned}
& C_{1}=\frac{q}{r+\lambda} \cdot\left(\frac{2 q}{\sigma^{2}}\right)^{k} \frac{\Gamma\left(\frac{1}{2}-k+m\right)}{\Gamma(1+2 m)} ; \\
& C_{2}=C_{1} ; \frac{\left(\frac{1}{2}+k+m\right) \operatorname{WhM}\left(k+1, m, \frac{2 q}{\sigma^{2}}\right)+\operatorname{WhM}\left(k, m, \frac{2 q}{\sigma^{2}}\right)}{\operatorname{WhW}\left(k+1, m, \frac{2 q}{\sigma^{2}}\right)-\operatorname{WhW}\left(k, m, \frac{2 q}{\sigma^{2}}\right)} ; \\
& D_{1}=0 ; \\
& D_{2}=\frac{\frac{1}{r+\lambda}}{\operatorname{WhW}\left(k+1, m, \frac{2 q}{\sigma^{2}}\right)-\operatorname{WhW}\left(k, m, \frac{2 q}{\sigma^{2}}\right)} .
\end{aligned}
$$




\subsection{Value of Cashflows at Inception (time 0)}

The values of liabilities and the normalized revenue streams for the GWB product at time $T$, when the withdrawals start will be given by

$$
\begin{aligned}
& L\left(C_{T}, B_{T}, T\right)=B_{T} \cdot l\left(\frac{C_{T}}{B_{T}}\right) ; \\
& G\left(C_{T}, B_{T}, T\right)=B_{T} \cdot g\left(\frac{C_{T}}{B_{T}}\right),
\end{aligned}
$$

where the functions $l(\cdot), g(\cdot)$ and the various parameters in the formulae are given in (17), (18) and (19)-(22). Since there are no cash outflows involved in phase 1 or during the waiting period, when the investor does not withdraw, it follows that, if $I_{t}$ denotes the indicator variable that the investor is alive at time $t$, then

$$
\begin{aligned}
L\left(C_{0}, B_{0}, 0\right) & =\mathbb{E}^{\mathbb{Q}}\left[I_{T} e^{-r T} L\left(C_{T}, B_{T}, T\right)\right] \\
& =e^{-r T} \mathbb{E}\left[I_{T}\right] \mathbb{E}^{\mathbb{Q}}\left[B_{T} l\left(\frac{C_{T}}{B_{T}}\right)\right] .
\end{aligned}
$$

For the revenue stream, we have

$$
\begin{aligned}
G\left(C_{0}, B_{0}, 0\right) & =\mathbb{E}^{\mathbb{Q}}\left[\int_{0}^{T} e^{-r t} I_{t} B_{t} d t+I_{T} e^{-r T} G\left(C_{T}, B_{T}, T\right)\right] \\
& =\int_{0}^{T}\left(e^{-r t} \cdot \mathbb{E}\left[I_{t}\right] \cdot \mathbb{E}^{\mathbb{Q}}\left[B_{t}\right]\right) d t+e^{-r T} \cdot \mathbb{E}\left[I_{T}\right] \cdot \mathbb{E}^{\mathbb{Q}}\left[B_{T} \cdot g\left(\frac{C_{T}}{B_{T}}\right)\right] .
\end{aligned}
$$

From (23) and (24), it follows that, to compute the value of the product at time 0, we need:

- The joint distribution of $B_{T}$ and $C_{T}$ under the risk neutral measure.

- The marginal distribution of $B_{t}$ under the risk neutral measure.

Fortunately, both of these quantities are computable, using properties of Brownian Motion. It is easier to work with the transformed processes

$$
\begin{aligned}
c_{t} & \triangleq \ln \left(\frac{C_{t}}{C_{0}}\right) ; \\
b_{t} & \triangleq \sup _{u: 0 \leq u \leq t} c_{u} \\
& =\ln \frac{\left(\sup _{u: 0 \leq u \leq t} C_{u}\right)}{C_{0}}=\ln \left(\frac{B_{t}}{B_{0}}\right) .
\end{aligned}
$$

The equality in (26) follows from the fact that $\ln (\cdot)$ is a monotonous function and that $C_{0}=B_{0}$.

Using the reflection principle and change of measures, it can be shown that the joint distribution of $c_{t}, b_{t}$ under $\mathbb{Q}$ is given by

$$
\begin{aligned}
f_{c_{t}, b_{t}}^{Q}(z, m) & =\frac{2(2 m-z)}{\sigma^{2} t} \cdot \frac{1}{\sigma \sqrt{t}} \Phi\left(\frac{2 m-z}{\sigma \sqrt{t}}\right) \cdot \exp \left(\frac{\nu}{\sigma^{2}} z-\frac{\nu^{2}}{\sigma^{2}} t\right) \ldots m \geq z, \\
\text { where } \nu & =r-\frac{1}{2} \sigma^{2} .
\end{aligned}
$$


The function $\Phi(x)=\frac{1}{\sqrt{2 \pi}} \exp \left(-\frac{x^{2}}{2}\right)$ denotes the standard normal density function. The marginal distribution of $b_{t}$ on the other hand is given by

$$
f_{b_{t}}^{Q}(m)=2 \cdot \frac{1}{\sigma \sqrt{t}} \Phi\left(\frac{m-\nu t}{\sigma \sqrt{t}}\right)-\frac{2 \nu}{\sigma^{2}} \exp \left(\frac{2 \nu m}{\sigma^{2}}\right) \cdot \mathrm{N}\left(\frac{-m-\nu t}{\sigma \sqrt{t}}\right) .
$$

Here $\mathrm{N}(\cdot)$ denotes the standard normal cumulative density function. The derivations which use the standard techniques involving the Reflection Principle in Brownian Motion and change of measures (see, for example, Karatzas and Shreve [21] for details) are presented in Appendix B for completeness.

These distributions then allow us to compute the expectations in (23) and (24) and consequently $L(C, B, 0)$ and $G(C, B, 0)$.

Note that (27) and (29) are respectively the joint distribution of $\left(b_{T}, c_{T}\right)$ and marginal distribution of $b_{t}$ conditional on $b_{0}=c_{0}$.

In this paper, we are primarily concerned with valuation of the GWB for life offering, i.e., the value at inception. If it is desired to evaluate the value of the product at an intermediate time $s$ during phase 1 , such that, $0<s<T$, we would need the joint distribution of $\left(b_{T}, c_{T}\right)$ conditional on general values of $b_{s}, c_{s}$ satisfying $c_{s} \leq b_{s}$. This can be in fact obtained readily from (27) and (29) by noting that

$$
\begin{aligned}
b_{t} & =\sup _{u: 0 \leq u \leq t} c_{u} \\
& =\max \left\{b_{s}, \sup _{u: s \leq u \leq t} c_{u}\right\} .
\end{aligned}
$$

Unfortunately, although (27) provides an explicit expression for the joint distribution of $B_{t}$ and $C_{t}$ through (27), the integrals in (23) and (24) do not have a closed form representation and must be evaluated numerically.

Finally, we emphasize that, we have assumed an exponential mortality model for the investor only for phase 2 . Investor mortality rates during phase 1, to be used in (23) and (24) can be arbitrary.

We now use the expressions derived in this section to compute the value and sensitivity of a typical GWB for life offering.

\section{Numerical Results}

In this section, we use the results derived in Section 3 for some numerical illustrations. As the model presented in Section 3 is an approximation of the actual GWB product, we focus more on risk and sensitivity analysis rather than absolute valuations. The experimental set-up is as follows: 


\section{Product Parameters}

- We set the minimum waiting period $W=3$ years, the retirement age $A_{R}=65$ years and the guaranteed withdrawal rate $q=6 \%$. These are indicative of typical offerings.

- For computing the net value of the GWB, we will assume that the fees are charged at the rate $h=0.65 \%$ of the benefit base and take a reference initial investment of $C_{0}=100$. Thus the net value figures that we obtain can be interpreted to have units of percentage of sales of VAs for which GWB for life was elected.

\section{Investors' Profiles}

- We consider the investor age at inception, i.e., $A$ to vary in the range 50 to 70 years.

- The investor chooses an asset-mix for her VA fund by selecting a target level of exposure (by value) that her portfolio will have to equities and we denote the same by $\alpha$. We consider four levels for $\alpha-20 \%, 40 \%, 60 \%$ and $80 \%$. The balance of the portfolio will be invested in relatively less volatile instruments, which we refer to as "'alternatives"'. The VA fund is also assumed to be continuously rebalanced to maintain the taget exposures.

- We would also like to consider the overall or average value of the GWB for the insurance company for sales across different investor cohorts and asset-mixes. For this, we assume that the distribution of client's age at inception $A$, weighted by her initial investment amount is uniform in the range 50-70. Further, for each cohort, the fraction $\alpha$ of investment in equities has values $20 \%, 40 \%, 60 \%$ and $80 \%$ with probabilities $0.1,0.4,0.4$ and 0.1 respectively. We refer to a portfolio of clients with the stated distribution of age and asset-mix selection as the "meta-portfolio" and this is again indicative of a typical VA client pool.

- For estimating mortality risk, we use the year 2008 mortality table published by the Pension Benefit Guaranty Corporation $(\mathrm{PGBC})^{7}$ and which is used to value annuities under Employee Retirement Income Security Act (ERISA) Section 4050. We convert this agewise table into a continuous time mortality rate function by modeling investor death as the arrival of a time inhomogeneous Poisson process with piecewise constant intensities, each piece being of length one year ${ }^{8}$. Then, for phase 2 , i.e., for $t>T$, for which the model in Section 3 assumes that the investor's residual life is exponentially distributed, we set the intensity to be the inverse of the expected residual life of the investor at $T$, as implied from the original mortality rate function.

7. Available online at http://www.pbgc.gov/practitioners/mortality-table/content/page15511.html.

8. This is commonly known as De-Moivre's approximation. 


\section{Asset Dynamics}

We assume that both equity markets as well as alternatives have log-normal returns and are uncorrelated. The Black Scholes volatility of the equity market returns $\sigma_{e}$, is set to $20 \%$ (annualized), while the same for the alternatives, $\sigma_{b}$, is set to $2 \%$ (annualized). The one year risk-free rate is taken to be $3.5 \%$. Because the VA fund is rebalanced continually, its fund index $S_{t}$ will follow a geometric Brownian motion consistent with the assumptions of Section 3. Its volatility $\sigma$ for an exposure $\alpha$ to equities is obtained as

$$
\sigma^{2}=\alpha^{2} \cdot \sigma_{e}^{2}+(1-\alpha)^{2} \cdot \sigma_{b}^{2}
$$

\section{Results}

Figure 1 shows how the break even fees and the net value to the insurance company (at $h=$ $0.65 \%$ fees) vary with cohort age for different asset mix choices. We observe that:
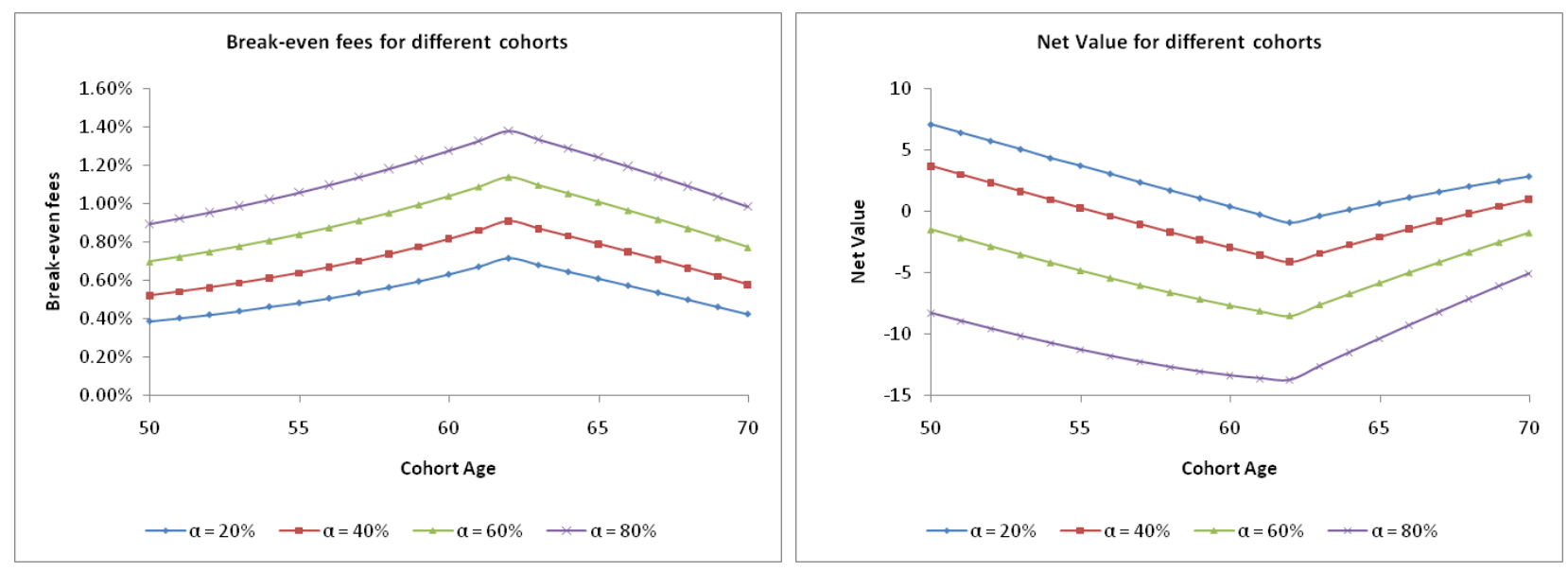

Fig. 1. Net Value as a function of Age.

- In general, the more aggressive the asset mix, the more expensive would be the GWB for life guarantee. A decomposition of the net-value reveals that both the payout liabilities (and so should capital requirements) and revenues increase sharply with volatilities. The corresponding changes in the net value and break-even fees are more subdued because the fee structure which is indexed to the benefit base provides a greater upside in revenues for more aggressive asset mixes and helps to somewhat offset the increases in the liabilities.

- Cohort age is a significant determinant of the value of the product. The ideal time for an investor to opt for GWB is probably just before retirement so as to minimize the number 
of years in phase 1 and maximize those during which the withdrawal guarantee applies, i.e., phase 2 .

We find that there is a significant risk of adverse selection of clientele in the one price fits all approach companies have been using to market and sell the GWB. In the long run, companies must differentiate in fees based on the investor age and their fund selections.

Next, we consider the sensitivity of the entire client portfolio, i.e., the meta-portfolio described earlier to shifts in interest rates and mortality rates. Figure 2 show how the break-even fees and the net-value fluctuate with the interest rate $r$. As expected, the GWB guarantee becomes more expensive as interest rates decrease. Further, the relationship between net value and interest rates is convex 9 . This indicates that interest rate volatility will work to lower GWB's valuations for insurance companies and must be considered in pricing it. Finally, we compute the net
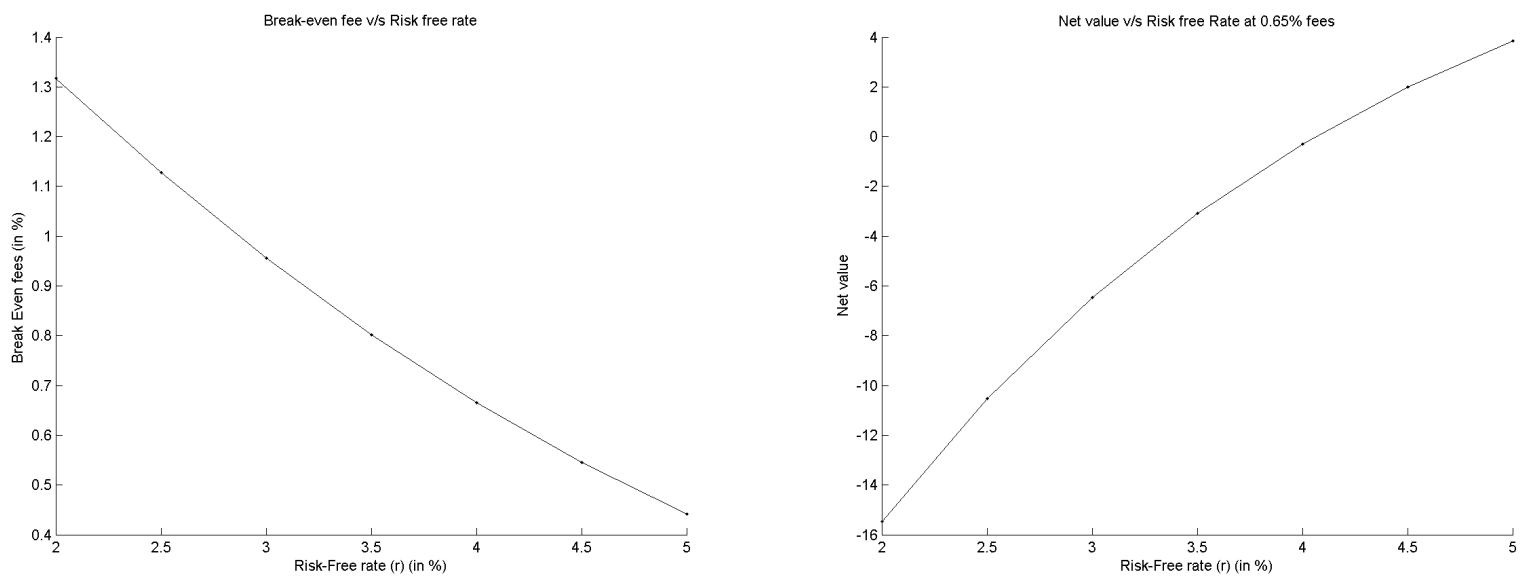

Fig. 2. Variation of break even fees and net-value with interest rate for the meta-portfolio.

value and break even fees for the meta-portfolio if all average residual lives (during phase 2 ) were to change by \pm 1 . The numbers, presented in Table 1 , show that the mortality related risk is substantial. To put things in perspective, the average residual lives at 65 for healthy males (18.74) and females (21.21) in the US differ by more than 2 years. Thus, the scenarios considered in Table 1 are quite plausible. Further, because there are no liquid mortality sensitive instruments, the resultant risk can be difficult to manage.

9. An intuitive reason for this convex nature is that as the risk-free rate decreases, not only does the possibility of a withdrawal shortfall increase but also the discount factor that would apply to the resulting payouts lowers. 


\begin{tabular}{c|cc}
\hline $\begin{array}{c}\text { Change in Avg. } \\
\text { Residual Life }\end{array}$ & Break even fee & Net Value at $h=0.65 \%$ \\
\hline 0 (Baseline) & $0.80 \%$ & -3.09 \\
+1 & $0.85 \%$ & -4.08 \\
-1 & $0.76 \%$ & -2.10 \\
\hline \hline
\end{tabular}

\section{TABLE 1}

Impact of changes in mortality rates on break even fees and net-value for the meta-portfolio.

\section{Pricing GWB using Alternate Models}

In this section, we seek to price the GWB for life in a more realistic setting. We consider the product specification as described in Section 2, with discrete (annual) step-ups and withdrawals and allow for an arbitrary distribution of retirement lifespans. We thus relax the two key simplifying assumptions made in the model of Section 3. The analysis in Section 3 showed that the interest rate volatility can impact GWB valuation. Also, GWB is like a deep out of the money option. In this section, we therefore consider models that allow interest rates to be stochastic and VA returns to have fatter tails and their implications on valuation.

As before, for a given sample path $\omega$, let $L_{n}\left(C_{n}, B_{n}, \omega\right)$ and $G_{n}\left(C_{n}, B_{n}, \omega\right)$ denote respectively the values of liabilities and the revenue base (i.e., revenues normalized by the fee rate $h$ ), at the $n^{\text {th }}$ anniversary, excluding the cash-flows pertaining to the $n^{\text {th }}$ anniversary. Additionally, we let $r_{n}^{f} \triangleq \int_{n-1}^{n} r_{s} d s$ denote the continuously compounded risk-free rate for the interval $(n-1, n]$ and $I_{n}$ be the indicator variable that the investor is alive at the end of year $n$. Then,

$$
\begin{aligned}
& I_{n}(\omega) L_{n}\left(C_{n}, B_{n}, \omega\right)=I_{n+1}(\omega) \cdot e^{-r_{n+1}^{f}}\left(\left(q_{n+1} B_{n}-C_{n} R_{n+1}^{s}\right)^{+}+L_{n+1}\left(C_{n+1}, B_{n+1}, \omega\right)\right) \\
& I_{n}(\omega) G_{n}\left(C_{n}, B_{n}, \omega\right)=I_{n+1}(\omega) \cdot e^{-r_{n+1}^{f}}\left(B_{n}+G_{n+1}\left(C_{n+1}, B_{n+1}, \omega\right)\right)
\end{aligned}
$$

For an investor aged $A$, we define

$$
\lambda_{n}^{A} \triangleq \ln \left(\frac{\mathbb{P}\left(I_{n}=1\right)}{\mathbb{P}\left(I_{n-1}=1\right)}\right) .
$$

$\lambda_{n}^{A}$ is thus the "hazard" or mortality rate function, assumed to be stationary. Thus, if $\lambda_{n}$ is the hazard rate of a population at birth then, $\lambda_{n}^{A}=\lambda_{A+n}$ and $\mathbb{P}\left(I_{n}=1\right)=\exp \left(-\sum_{i=1}^{n} \lambda_{i}^{A}\right)$. We assume there exists a finite $\bar{N}$ such that $\mathbb{P}\left(I_{\bar{N}+1}=1\right)=0$, or equivalently, $\lambda_{\bar{N}+1}=\infty$. Let $L_{n}\left(C_{n}, B_{n}\right)$ and $G\left(C_{n}, B_{n}\right)$ respectively denote the fair value of liabilities and revenues conditional on the investor being alive at year $n$. 
From (1) and (31), it follows that all cash-flows and state variable updates or "epochs" occur at GWB anniversaries. Then, any asset dynamics model that gives the joint distribution of one year risk free rate $r_{n}^{f}$ and $R_{n}^{s}$ can be used to price the GWB. Further, if this model is Markovian with state variable $Y_{n}$ and the investor always withdraws the contract stipulated amount $q_{n+1} B_{n}$ at the $n^{\text {th }}$ anniversary, then

$$
\begin{aligned}
L_{n}\left(C_{n}, B_{n}, Y_{n}\right)= & e^{-\lambda_{n+1}^{A}} \mathbb{E}^{\mathbb{Q}}\left[e ^ { - r _ { n + 1 } ^ { f } } \left\{\left(q_{n+1} B_{n}-C_{n} R_{n+1}^{s}\right)^{+}\right.\right. \\
& \left.\left.+L_{n+1}\left(\left(C_{n} R_{n+1}^{s}-q_{n+1} B_{n}\right)^{+}, \max \left(B_{n}, C_{n} R_{n+1}^{s}-q_{n+1} B_{n}\right), Y_{n+1}\right)\right\} \mid Y_{n}\right], \\
G_{n}\left(C_{n}, B_{n}, Y_{n}\right)= & e^{-\lambda_{n+1}^{A}} \mathbb{E}^{\mathbb{Q}}\left[e^{-r_{n+1}^{f}}\{1\right. \\
& \left.\left.+G_{n+1}\left(\left(C_{n} R_{n+1}^{s}-q_{n+1} B_{n}\right)^{+}, \max \left(B_{n}, C_{n} R_{n+1}^{s}-q_{n+1} B_{n}\right), Y_{n+1}\right)\right\} \mid Y_{n}\right] .
\end{aligned}
$$

As before, we assume that $R_{n}^{s}$ is obtained as a blend of returns on equities and returns on less volatile alternative investment instruments. We will assume that, for the alternative investments, the excess returns over the risk free rate $r_{n}^{f}$ follows a geometric Brownian motion. For modeling interest rates and equity market returns, we consider three different models:

- BSM model: In this model the interest rates $r_{n}^{f}$ are taken to be constant at $r$ and the equity market returns to be log-normal. Since the alternative instuments' returns are also lognormal and the VA fund is continuously rebalanced, it will also have log-normal returns.

- SILN model: In this model, we allow for interest rates to be stochastic and use a two factor Vasicek model, which is an extension of the model proposed by Vasicek [29] to model the short rate. We continue to assume log-normal returns for equities and by extension for the VA fund.

- SISV model: Here, the interest rates are modeled by a two-factor Vasicek model as in the SILN model. In addition, we use the Heston model (see Heston [19]), which is a stochastic volatility model to model the excess of equity returns over the risk free rate.

We do not consider the effect of jumps in asset prices. We do not believe this to be significant as GWB is a long duration product and the jump effects are likely to be diffused out over the interval between two epochs. Also, jump models are difficult to calibrate in practice ${ }^{10}$.

These models do not yield to analytical solutions and GWB must be priced using Monte Carlo simulations or by numerically solving the difference equations implied by (32). Before

10. Chen, Vetzal and Forsyth [14] report that jumps significantly increase the product price for the GMWB. However, they add jumps on top of the diffusion process and do not indicate how much of the price increase is induced by an increase in the effective volatility of the diffusion process and how much can be attributed to purely the jump effects. 
we proceed to the valuation of the GWB, we furnish some more details about each of the three models proposed above.

\subsection{Black Scholes Asset Dynamics - BSM Model}

The BSM model is essentially the same as the continuous time model in Section 3, except that the step-ups are now annual and that the mortality process is general. For compliant investor withdrawals, the homogeneity relation of Proposition 1 for liabilities and revenue streams holds in the discrete time setting as well ${ }^{11}$. Also there is no state variable $Y_{n}$ in the Black-Scholes asset dynamics. Hence, we can define functions $l_{n}(\cdot)$ and $g_{n}(\cdot)$ that satisfy

$$
\begin{aligned}
& L_{n}\left(C_{n}, B_{n}\right)=B_{n} \cdot L_{n}\left(\frac{C_{n}}{B_{n}}, 1\right)=B_{n} \cdot l_{n}\left(\frac{C_{n}}{B_{n}}\right) \\
& G_{n}\left(C_{n}, B_{n}\right)=B_{n} \cdot G_{n}\left(\frac{C_{n}}{B_{n}}, 1\right)=B_{n} \cdot g_{n}\left(\frac{C_{n}}{B_{n}}\right) .
\end{aligned}
$$

Using (32), then for $x: 0 \leq x \leq 1$ :

$$
\begin{aligned}
& l_{n}(x)=e^{-\left(\lambda_{n+1}^{A}+r\right)} \cdot\left\{x \cdot \int_{-\infty}^{\ln \left(\frac{q_{n+1}}{x}\right)}\left(\frac{q_{n+1}}{x}-e^{z}\right) \cdot \Phi(z ; \mu, \sigma) d z\right. \\
& +\int_{\ln \left(\frac{q_{n+1}}{x}\right)}^{\ln \left(\frac{1+q_{n+1}}{x}\right)} l_{n+1}\left(x e^{z}-q_{n+1}\right) \cdot \Phi(z ; \mu, \sigma) d z \\
& \left.+x \cdot l_{n+1}(1) \cdot \int_{\ln \left(\frac{1+q_{n+1}}{x}\right)}^{\infty}\left(e^{z}-\frac{q_{n+1}}{x}\right) \cdot \Phi(z ; \mu, \sigma) d z\right\} ; \\
& g_{n}(x)=e^{-\left(\lambda_{n+1}^{A}+r\right)} \cdot\left\{1+\int_{\ln \left(\frac{q_{n+1}}{x}\right)}^{\ln \left(\frac{1+q_{n+1}}{x}\right)} g_{n+1}\left(x e^{z}-q_{n+1}\right) \cdot \Phi(z ; \mu, \sigma) d z\right. \\
& \left.+x \cdot g_{n+1}(1) \cdot \int_{\ln \left(\frac{1+q_{n+1}}{x}\right)}^{\infty}\left(e^{z}-\frac{q_{n+1}}{x}\right) \cdot \Phi(z ; \mu, \sigma) d z\right\} .
\end{aligned}
$$

Here, $\sigma$ is the volatility of the VA fund returns and $\mu \triangleq r-\frac{1}{2} \sigma^{2}$. Also $\Phi(z ; \mu, \sigma)$ denotes the normal density function for mean $\mu$ and variance $\sigma^{2}$. Since $l_{\bar{N}}(x)=g_{\bar{N}}(x)=0$ for all $x \in[0,1]$, one can obtain the values of the functions $l_{n}(\cdot)$ and $g_{n}(\cdot)$ from (35) and (36) using backward substitution. In practice, we can use a polynomial interpolation method to successively approximate $l_{n}(\cdot)$ and $g_{n}(\cdot)$, without evaluating any integrals involved numerically, as the moments of the normal distribution are available in a closed form.

For numerical computations, as before, we take one year risk-free rate to be $3.5 \%$, equity volatility $\sigma_{e}=20 \%$, alternative investments' volatility $\sigma_{b}=2 \%$ and calculate $\sigma$ using (30).

11. Note that this is actually a model independent property and can be proved using essentially the same arguments as in Proposition 1. 


\subsection{Stochastic Interest Rates and Lognormal Equity Returns - SILN Model}

For the SILN model, we use a 2-factor Vasicek Model for the short-rate ${ }^{12}$, as suggested in Chu and Kwok [15] for pricing Guaranteed Annuity Options (GAO). This short rate model (under the risk-neutral measure) is given by

$$
\begin{aligned}
r_{t} & =x_{t}^{1}+x_{t}^{2}+b(t) \\
d x_{t}^{1} & =-\kappa_{1} x_{t}^{1}+\sigma_{1} d Z_{t}^{1} \\
d x_{t}^{2} & =-\kappa_{2} x_{t}^{2}+\sigma_{1} d Z_{t}^{2} \\
<d Z_{t}^{1} \cdot d Z_{t}^{2}> & =\rho d t
\end{aligned}
$$

The function $b(t)$ is deterministic and chosen so as to agree with the initially observed yield curve in the market. Our model for returns on the equity markets index $E_{t}$ is

$$
\frac{d E_{t}}{E_{t}}=r_{t} d t+\sigma_{e} d Z_{t}^{Q}
$$

where $Z_{t}^{Q}$, is assumed to be independent of the factors driving the short rate. Thus, in essence we assume that the excess equity and alternative investments' returns (over the risk-free rate) are independent of the short rate process and are log-normal. As a result excess VA fund returns will also be lognormal. For the SILN model, the state variable $Y_{n}$ is the two dimensional vector $\left(x_{1, n}, x_{2, n}\right)$. We use (32) to value GWB directly through Monte-Carlo simulations. The short rate model considered here is a Gaussian model, and hence entails a small but non-zero probability for the short-rate to become negative. A way to avoid this problem is to instead use the CoxIngersoll-Ross process for short rates ${ }^{13}$, see Cox, Ingersoll and Ross, [16]. The Gaussian model however has the advantage that the state variable $Y_{n}$ and one year risk free factor $r_{n}^{f}$ are jointly normal when conditioned on $Y_{n-1}$. This allows us to use time steps as large as one year in the Monte Carlo simulations used to price the GWB and makes the computation much faster. For pricing the GWB, we use the structural-parameter values as specified in Chu and Kwok [15] viz., $\kappa_{1}=0.77, \kappa_{2}=0.08, \sigma_{1}=2 \%, \sigma_{2}=1 \%, \rho=-0.7$. We also adjust $b(t)$ so as to obtain an intial yield curve to be flat at $3.50 \%$ so as to make valuations obtained through this model comparable to the BSM model. The volatility in interest rates would increase the effective annual volatility in VA returns. Hence we adjust downwards the volatilities $\sigma_{e}$ and $\sigma_{b}$ of the excess equity and

12. We prefer a 2-factor model over the simpler one factor interest rate models, because in a one factor model, all forward interest rates become fully correlated.

13. For more details about the Vasicek and other interest rate models, we refer the reader to Duffie [18]. 
alternative investments' returns respectively so that the total volatility in one year equity and alternative investments' returns is the same as that in the case of the BSM model. Both the size and impact of this adjustment turn out to be negligible in the case of equity returns.

\subsection{Stochastic Interest Rates and Stochastic Volatility - SISV Model}

In the SISV model, we take both interest rates and equity volatilities to be stochastic. We continue to model the excess alternative investments' returns as lognormal and independent of both the excess equity returns as well as the interest rates. For interest rates, we use the same 2-factor Gaussian Model as described by (37) for the SILN model. We model excess equity market returns to follow the Heston process (see Heston [19]) under the risk neutral measure. Thus if $E_{t}$ represents the equity market index,

$$
\begin{aligned}
\frac{d E_{t}}{E_{t}} & =r_{t} d t+\sqrt{V_{t}} d Z_{t}^{Q} ; \\
d V_{t} & =\kappa_{s}\left(\theta_{s}-V_{t}\right)+\xi \sqrt{V_{t}} d Z_{t}^{\sigma} ; \\
<d Z_{t}^{Q} \cdot d Z_{t}^{\sigma}> & =\rho_{e} d t .
\end{aligned}
$$

Since the interest rate dynamics for the SISV model are the same as in SILN model, we continue to use the same parameters for them as in the SILN model. For the structural parameters in the Heston model in (39), we use most values close to those found by Bakshi, Cao and Chen in [3] by a time-series and cross-sectional analysis of options data. In particular, we take $\kappa_{s}=1, \xi=0.42, \rho_{e}=-0.75$. After fixing these parameters, we impose the constraint $V_{0}=\theta$, and simultaneously adjust them so that the Black-Scholes implied volatility for a 1-year ATM equity option, assuming a fixed one year interest rate of $3.5 \%$ is $20 \%$. This yields a value of $\theta=V_{0}=0.0496$. We do not adjust the excess equity return process to offset the increase in effective volatility of total equity returns due to interest rate volatilities here, but as discussed in the context of SILN model, do not expect this effect to be significant. We do compensate down the volatility of excess alternate investment returns to make the one year volatility of total returns the same as in the BSM model.

Appendix $\mathrm{C}$ briefly outlines the details associated with computation and possible optimizations in the numerical valuations of GWB for life using these three models.

\section{Numerical Results for GWB Valuation}

In this section, we discuss the results obtained from a numerical valuation of the GWB for life under the BSM, SILN and SISV models presented in Section 5. We use the same parameter 
values for the GWB product, investor profiles and mortality rates as in Section 4, except that we no longer assume mortality rates in phase 2 to be exponential. Table 2 shows the break-even

\begin{tabular}{|c|c|c|c|c|c|c|c|}
\hline \multirow[b]{2}{*}{$\begin{array}{l}\text { Cohort } \\
\text { Age }\end{array}$} & \multirow[b]{2}{*}{$\begin{array}{l}\text { Equity Ex- } \\
\text { posure }\end{array}$} & \multicolumn{3}{|c|}{ Break-even fees } & \multicolumn{3}{|c|}{ Net value at $h=0.65 \%$} \\
\hline & & BSM & SILN & SISV & BSM & SILN & SISV \\
\hline \multirow{4}{*}{50} & $20 \%$ & $0.16 \%$ & $0.26 \%$ & $0.27 \%$ & 13.47 & 10.90 & 10.52 \\
\hline & $40 \%$ & $0.29 \%$ & $0.39 \%$ & $0.42 \%$ & 10.17 & 7.63 & 6.63 \\
\hline & $60 \%$ & $0.49 \%$ & $0.57 \%$ & $0.62 \%$ & 4.86 & 2.42 & 0.97 \\
\hline & $80 \%$ & $0.71 \%$ & $0.78 \%$ & $0.83 \%$ & -2.03 & -4.36 & -5.88 \\
\hline \multirow{4}{*}{55} & $20 \%$ & $0.19 \%$ & $0.30 \%$ & $0.32 \%$ & 10.41 & 7.98 & 7.66 \\
\hline & $40 \%$ & $0.35 \%$ & $0.45 \%$ & $0.49 \%$ & 7.05 & 4.78 & 3.98 \\
\hline & $60 \%$ & $0.57 \%$ & $0.66 \%$ & $0.70 \%$ & 1.93 & -0.19 & -1.21 \\
\hline & $80 \%$ & $0.81 \%$ & $0.89 \%$ & $0.92 \%$ & -4.48 & -6.49 & -7.40 \\
\hline \multirow{4}{*}{60} & $20 \%$ & $0.24 \%$ & $0.36 \%$ & $0.37 \%$ & 7.41 & 5.35 & 5.15 \\
\hline & $40 \%$ & $0.43 \%$ & $0.53 \%$ & $0.55 \%$ & 4.14 & 2.35 & 1.84 \\
\hline & $60 \%$ & $0.67 \%$ & $0.75 \%$ & $0.78 \%$ & -0.47 & -2.09 & -2.70 \\
\hline & $80 \%$ & $0.93 \%$ & $1.00 \%$ & $1.02 \%$ & -6.01 & -7.53 & -7.99 \\
\hline \multirow{4}{*}{65} & $20 \%$ & $0.17 \%$ & $0.26 \%$ & $0.27 \%$ & 7.20 & 5.89 & 5.71 \\
\hline & $40 \%$ & $0.34 \%$ & $0.41 \%$ & $0.44 \%$ & 4.90 & 3.74 & 3.26 \\
\hline & $60 \%$ & $0.56 \%$ & $0.62 \%$ & $0.66 \%$ & 1.57 & 0.51 & -0.16 \\
\hline & $80 \%$ & $0.79 \%$ & $0.85 \%$ & $0.89 \%$ & -2.52 & -3.52 & -4.17 \\
\hline \multirow{4}{*}{70} & $20 \%$ & $0.06 \%$ & $0.12 \%$ & $0.13 \%$ & 7.49 & 6.88 & 6.74 \\
\hline & $40 \%$ & $0.17 \%$ & $0.22 \%$ & $0.25 \%$ & 6.43 & 5.82 & 5.32 \\
\hline & $60 \%$ & $0.32 \%$ & $0.37 \%$ & $0.43 \%$ & 4.57 & 3.96 & 3.13 \\
\hline & $80 \%$ & $0.51 \%$ & $0.55 \%$ & $0.62 \%$ & 2.02 & 1.43 & 0.41 \\
\hline Meta & Meta & $0.47 \%$ & $0.55 \%$ & $0.58 \%$ & 3.75 & 2.11 & 1.40 \\
\hline
\end{tabular}

TABLE 2

Break-even fees and net value for GWB under different models.

fees and the net value at $0.65 \%$ fees for various combinations of investor age at inception, $A$ and asset-mix selection, $\alpha$. As compared to the continuous step-ups and exponential retirement lifespan model, the break even fees are much lower and the net value much higher. This can be primarily attributed to annual rather than continuous step-ups. We observe that:

- Across all models, break-even fees and net-value numbers are significantly different for different asset mixes and cohort ages. This confirms the findings of Section 4 that charging a uniform premium across all cohorts and asset-mixes might lead to a selection bias in the 
investor pool. The GWB guarantee is more expensive for a more aggressive choice of the asset mix. Also consistent with Section 4, we find that under all models GWB becomes more expensive as the number of phase 2 years increase or as the number of phase 1 years decreases $^{14}$.

- Accounting for interest rate stochasticity (SILN model) substantially reduces valuations over the BSM model. The break-even fee for the composite or meta-portfolio increases from 47 basis points under the BSM model to 55 basis points under the SILN model, while the net value decreases from $3.75 \%$ of sales for BSM to $2.11 \%$ of sales for SILN.

- Incorporating stochastic volatility, through the SISV model leads to a further significant decrease in valuations over the SILN model. These reductions are again uniform across all cohorts and asset mix selections.

A breakdown of net-revenues, as shown in Table 3 reveals that the change in valuations under different models is driven primarily by an increase in the liabilities of the insurance company. This means that the choice of the model used to account for GWB will not only impact reported bottom-lines but also capital requirements for risk management.

Finally, we try to obtain a sense of the mortality related risk. For this we evaluate GWB for life under two alternate mortality rates:

- Mortality rates shifted to the right by one year ${ }^{15}$ to get the new rates as $\lambda_{n}^{-}=\lambda_{n+1}$, with $\lambda_{m}^{-}=\infty$ for $m \geq \bar{N}$.

- Mortality rates shifted to the left by one year to get the new rates as $\lambda_{n}^{+}=\lambda_{n-1}$.

Table 4 lists the corresponding values of break even fees and net values. This confirms the finding of Section 4 that there is considerable risk due to realized investor pool longevities.

\section{Conclusion}

In this paper, we examined the GWB for life, an exotic option that many insurance companies are offering with VA products. We first obtained an analytical solution for the GWB by employing some simplifying assumptions. This helped us to draw insights about the possible risk factors. We then repriced GWB using realistic models and without the simplifying assumptions. All three valuation models used for this purpose were tuned so that they would imply consistent levels of interest rates and option implied volatility of asset returns. We find that:

14. It peaks for investors who are aged 62, when opening the account.

15. This operation leads to a decrease in longevities. 


\begin{tabular}{|c|c|c|c|c|c|c|c|}
\hline \multirow[b]{2}{*}{$\begin{array}{l}\text { Cohort } \\
\text { Age }\end{array}$} & \multirow[b]{2}{*}{$\begin{array}{l}\text { Equity } \\
\text { Exposure }\end{array}$} & \multicolumn{3}{|c|}{ Payout Values } & \multicolumn{3}{|c|}{ Revenues at $h=0.65 \%$ fees } \\
\hline & & BSM & SILN & SISV & BSM & SILN & SISV \\
\hline \multirow{4}{*}{50} & $20 \%$ & 4.25 & 7.07 & 7.58 & 17.72 & 17.97 & 18.08 \\
\hline & $40 \%$ & 8.43 & 11.25 & 12.44 & 18.60 & 18.88 & 19.06 \\
\hline & $60 \%$ & 15.02 & 17.74 & 19.35 & 19.88 & 20.16 & 20.32 \\
\hline & $80 \%$ & 23.43 & 26.03 & 27.62 & 21.40 & 21.67 & 21.74 \\
\hline \multirow{4}{*}{55} & $20 \%$ & 4.28 & 6.94 & 7.35 & 14.69 & 14.93 & 14.99 \\
\hline & $40 \%$ & 8.38 & 10.89 & 11.78 & 15.43 & 15.67 & 15.75 \\
\hline & $60 \%$ & 14.53 & 16.88 & 17.94 & 16.46 & 16.69 & 16.72 \\
\hline & $80 \%$ & 22.12 & 24.35 & 25.21 & 17.65 & 17.86 & 17.81 \\
\hline \multirow{4}{*}{60} & $20 \%$ & 4.35 & 6.59 & 6.82 & 11.75 & 11.94 & 11.95 \\
\hline & $40 \%$ & 8.20 & 10.18 & 10.68 & 12.34 & 12.52 & 12.51 \\
\hline & $60 \%$ & 13.58 & 15.37 & 15.91 & 13.11 & 13.28 & 13.21 \\
\hline & $80 \%$ & 19.99 & 21.66 & 21.97 & 13.98 & 14.13 & 13.98 \\
\hline \multirow{4}{*}{65} & $20 \%$ & 2.55 & 3.98 & 4.17 & 9.74 & 9.87 & 9.87 \\
\hline & $40 \%$ & 5.31 & 6.59 & 7.03 & 10.21 & 10.33 & 10.28 \\
\hline & $60 \%$ & 9.23 & 10.40 & 10.97 & 10.80 & 10.91 & 10.81 \\
\hline & $80 \%$ & 13.96 & 15.08 & 15.56 & 11.44 & 11.55 & 11.39 \\
\hline \multirow{4}{*}{70} & $20 \%$ & 0.79 & 1.49 & 1.63 & 8.29 & 8.37 & 8.37 \\
\hline & $40 \%$ & 2.22 & 2.91 & 3.38 & 8.65 & 8.73 & 8.70 \\
\hline & $60 \%$ & 4.55 & 5.23 & 5.98 & 9.11 & 9.19 & 9.11 \\
\hline & $80 \%$ & 7.60 & 8.26 & 9.15 & 9.62 & 9.69 & 9.56 \\
\hline Meta & Meta & 9.58 & 11.40 & 12.10 & 13.33 & 13.51 & 13.50 \\
\hline
\end{tabular}

TABLE 3

Breakdown of GWB for life liabilities and revenues for the insurance company under different models.

1) On the whole, a fee of 65 basis points appears enough to cover the cost of the guarantee, though this rests on the assumptions made about the distribution of investor profiles.

2) There is insufficient price discrimination and GWB for life can be priced at below par value for the riskiest of segments, i.e., clients with imminent retirement and choosing an aggressive investment style. The product needs to be either re-engineered or pricediscriminated based on age and investment styles to avoid an adverse selection risk.

3) Accounting for interest rate volatility and using models that imply fatter tails for fund return distributions can substantially increase the cost of the GWB for the company. 


\begin{tabular}{r|c|ccc} 
& & \multicolumn{4}{|c}{ Mortality rates } \\
\hline & Model & $\lambda_{n}$ & $\lambda_{n}^{+}$ & $\lambda_{n}^{-}$ \\
\hline \multirow{3}{*}{ Break-even fees } & BSM & $0.47 \%$ & $0.51 \%$ & $0.43 \%$ \\
& SILN & $0.55 \%$ & $0.59 \%$ & $0.50 \%$ \\
& SISV & $0.58 \%$ & $0.63 \%$ & $0.54 \%$ \\
\hline \multirow{3}{*}{ Net value at $\mathbf{h}=\mathbf{0 . 6 5} \%$} & BSM & 3.75 & 2.96 & 4.47 \\
& SILN & 2.11 & 1.19 & 2.96 \\
& SISV & 1.40 & 0.52 & 2.23 \\
\hline \hline
\end{tabular}

TABLE 4

Sensitivity of the meta portfolio value to mortality rates under different models.

4) Valuation of GWB has a high level of dependence on model choice. All three models considered here - BSM, SILN and SISV can be considered, in principle, as reasonable models for pricing GWB, especially since there are no comparable securities of such duration traded in the markets. Choosing one model over other can change valuation by more than $2 \%$ of sales, or for a typical insurance company selling $\$ 10$ billion of VAs annually, by about $\$ 200$ million a year. The value, especially liabilities, from the product will not be realized for decades and hence the pricing ambiguity poses a difficulty for investors. For regulators too, the considerably different levels of financial risk and liabilities suggested by different models can create an ambiguity in setting the requisite capitalization levels. We believe that regulatory and accounting bodies should recommend a standardized model for pricing GWB like guarantees.

5) The GWB for life guarantees are also very sensitive to the investor pool longevity. This coupled with the model risk implies that they constitute a significant long-term risk that can be extremely challenging to hedge.

\section{APPENDIX A}

\section{WHITTAKER FUNCTIONS AND SOME BASIC PROPERTIES}

The function $\operatorname{WhM}(k, m, z)$ can be defined in terms of hypergeometric function (see Mathworld [23]) as

$$
\operatorname{WhM}(k, m, z)=\exp \left(-\frac{z}{2}\right) z^{m+\frac{1}{2}}{ }_{1} F_{1}\left(\frac{1}{2}+m-k, 1+2 m, z\right) .
$$


${ }_{1} F_{1}(a, b, z)$ denotes a confluent hypergeometric function of the first kind. It has an integral representation

$$
{ }_{1} F_{1}(a, b, z)=\frac{\Gamma(b)}{\Gamma(b-a) \Gamma(a)} \int_{0}^{1} \exp (z . t) t^{a-1}(1-t)^{b-a-1} d t .
$$

Proposition 3: Let

$$
f_{1}(x) \triangleq x^{-k} e^{-\frac{a}{2 x}} \operatorname{WhM}\left(k, m, \frac{a}{x}\right)
$$

where $a>0$. Then,

$$
\begin{aligned}
\lim _{x \rightarrow 0^{+}} f_{1}(x) & =a^{-k} \frac{\Gamma(1+2 m)}{\Gamma\left(\frac{1}{2}-k+m\right)} \\
f_{1}^{\prime}(x) & =-\left(\frac{1}{2}+k+m\right) x^{-(k+1)} e^{-\frac{a}{2 x}} \operatorname{WhM}\left(k+1, m, \frac{a}{x}\right) .
\end{aligned}
$$

Proof:

$$
\begin{aligned}
\lim _{x \rightarrow 0^{+}} f_{1}(x) & =\lim _{x \rightarrow 0^{+}} a^{-k}\left(\frac{a}{x}\right)^{\frac{1}{2}+k+m} e^{-\frac{a}{x}}{ }_{1} F_{1}\left(\frac{1}{2}-k+m, 1+2 m, \frac{a}{x}\right) \\
& =a^{-k} \lim _{z \rightarrow \infty} z^{\frac{1}{2}+k+m} e^{-z}{ }_{1} F_{1}\left(\frac{1}{2}-k+m, 1+2 m, z\right) \\
& =a^{-k} \lim _{z \rightarrow \infty} z^{\frac{1}{2}+m+k} e^{z} \frac{\Gamma(1+2 m)}{\Gamma\left(\frac{1}{2}-k+m\right) \Gamma\left(\frac{1}{2}+k+m\right)} \cdot \int_{0}^{1} e^{z t} t^{-\frac{1}{2}-k+m}(1-t)^{-\frac{1}{2}+k+m} d t \\
& =a^{-k} \frac{\Gamma(1+2 m)}{\Gamma\left(\frac{1}{2}-k+m\right) \Gamma\left(\frac{1}{2}+k+m\right)} \cdot \\
& =\lim _{z \rightarrow \infty} \int_{0}^{-k} \frac{\Gamma(1+2 m)}{\Gamma\left(\frac{1}{2}-k+m\right) \Gamma\left(\frac{1}{2}+k+m\right)} \lim _{z \rightarrow \infty} \int_{0}^{1} e^{-z t} t^{-\frac{1}{2}+k+m}(1-t)^{-\frac{1}{2}-k+m} z^{k+\frac{1}{2}+m} d t \\
& =a^{-k} \frac{\Gamma(1+2 m)}{\Gamma\left(\frac{1}{2}-k+m\right) \Gamma\left(\frac{1}{2}+k+m\right)} \lim _{z \rightarrow \infty} \int_{0}^{z} e^{-y} y^{-\frac{1}{2}+k+m}\left(1-\frac{y}{z}\right)^{-\frac{1}{2}-k+m} d y \\
& =a^{-k} \frac{\Gamma(1+2 m)}{\Gamma\left(\frac{1}{2}-k+m\right) \Gamma\left(\frac{1}{2}+k+m\right)} \lim _{z \rightarrow \infty} \int_{0}^{z} e^{-y} y^{-\frac{1}{2}+k+m} d y \\
& =a^{-k} \frac{\Gamma(1+2 m)}{\Gamma\left(\frac{1}{2}-k+m\right)} .
\end{aligned}
$$

The property (41) was obtained by symbolic differentiation using Maple 9.

The function $\mathrm{WhW}(k, m, z)$ has the following integral representation (see Mathworld [23]):

$$
\operatorname{WhW}(k, m, z)=\frac{e^{-\frac{z}{2}} z^{k}}{\Gamma\left(\frac{1}{2}-k+m\right)} \int_{0}^{\infty} t^{-\frac{1}{2}-k+m}\left(1+\frac{t}{z}\right)^{-\frac{1}{2}+k+m} e^{-t} d t
$$

Proposition 4: Let

$$
f_{2}(x) \triangleq x^{-k} e^{-\frac{a}{2 x}} \mathrm{WhW}\left(k, m, \frac{a}{x}\right)
$$


where $a>0$ and $m-k-\frac{1}{2}>-1$. Then,

$$
\begin{aligned}
\lim _{x \rightarrow 0^{+}} f_{2}(x) & =0 ; \\
f_{2}^{\prime}(x) & =x^{-(k+1)} e^{-\frac{a}{2 x}} \mathrm{WhW}\left(k+1, m, \frac{a}{x}\right) .
\end{aligned}
$$

Proof:

$$
\begin{aligned}
\lim _{x \rightarrow 0^{+}} f_{2}(x) & =\lim _{x \rightarrow 0^{+}} a^{-k} \frac{\left(\frac{a}{x}\right)^{2 k} e^{-\frac{a}{x}}}{\Gamma\left(\frac{1}{2}-k+m\right)} \int_{0}^{\infty} t^{-\frac{1}{2}-k+m}\left(1+\frac{t x}{a}\right)^{-\frac{1}{2}+k+m} e^{-t} d t \\
& =\frac{a^{-k}}{\Gamma\left(\frac{1}{2}-k+m\right)} \lim _{z \rightarrow \infty} z^{2 k} e^{-z} \int_{0}^{\infty} t^{-\frac{1}{2}-k+m}\left(1+\frac{t}{z}\right)^{-\frac{1}{2}+k+m} e^{-t} d t \\
& =\frac{a^{-k}}{\Gamma\left(\frac{1}{2}-k+m\right)}\left(\lim _{z \rightarrow \infty} z^{2 k} e^{-z}\right) \cdot\left(\lim _{z \rightarrow \infty} \int_{0}^{\infty} t^{-\frac{1}{2}-k+m}\left(1+\frac{t}{z}\right)^{-\frac{1}{2}+k+m} e^{-t} d t\right) \\
& =0 .
\end{aligned}
$$

The property (44) was again obtained by symbolic differentiation using Maple 9.

\section{APPENDIX B}

\section{JOINT DISTRIBUTION OF $B_{t}$ AND $C_{t}$}

Consider the following normalizations of the process $c_{t}$ and $b_{t}$ defined in (25) and (26):

$$
\begin{aligned}
& y_{t}=\frac{c_{t}}{\sigma}=\frac{1}{\sigma} \ln \left(C_{t}\right) ; \\
& u_{t}=\frac{b_{t}}{\sigma}=\frac{1}{\sigma} \ln \left(B_{t}\right) .
\end{aligned}
$$

Applying Ito's lemma to (3), we get

$$
\begin{aligned}
d y_{t} & =\left(\frac{r}{\sigma}-\frac{1}{2} \sigma\right) d t+d Z_{t}^{Q}, \\
\text { i.e., } y_{t} & =\eta t+Z_{t}^{Q}, \\
\text { where } \eta & =\frac{r}{\sigma}-\frac{1}{2} \sigma .
\end{aligned}
$$

Then, by Girsanov's theorem, $y_{t}$ is a Brownian Motion under the measure $R$ whose RadonNikodym derivative is given by

$$
\frac{d Q}{d R}=\exp \left(\eta y_{t}-\frac{1}{2} \eta^{2} t\right)
$$

Since $y_{t}$, is a Brownian motion under $R$ and $u_{t}$ its supremum, using the well-known reflection principle (see Karatzas and Shreve [21]), we get: 


$$
\begin{aligned}
& \mathbb{P}^{R}\left(y_{t} \leq u-a, u_{t} \geq u\right)= \begin{cases}\mathbb{P}^{R}\left(y_{t} \geq u+a\right) & \ldots, \quad a \geq 0 ; \\
\mathbb{P}^{R}\left(u_{t} \geq u\right)-\mathbb{P}^{R}\left(y_{t}>u-a, u_{t} \geq u\right) & \\
\quad=\mathbb{P}^{R}\left(u_{t} \geq u\right)-\mathbb{P}^{R}\left(y_{t}>u-a\right) \quad \ldots, \quad a<0 .\end{cases} \\
& \text { i.e., } \mathbb{P}^{R}\left(y_{t} \leq y, u_{t} \geq u\right)=\left\{\begin{array}{c}
\mathbb{P}^{R}\left(y_{t} \geq 2 u-y\right) \\
=1-\mathrm{N}\left(\frac{2 u-y}{\sqrt{t}}\right) \\
\mathbb{P}^{R}\left(u_{t} \geq u\right)-\mathbb{P}^{R}\left(y_{t}>y, u_{t} \geq u\right) \\
\quad=\mathbb{P}^{R}\left(u_{t} \geq u\right)-\left(1-\mathrm{N}\left(\frac{y}{\sqrt{t}}\right)\right) \ldots, \quad y \leq u ;
\end{array}\right.
\end{aligned}
$$

Taking derivatives, we get:

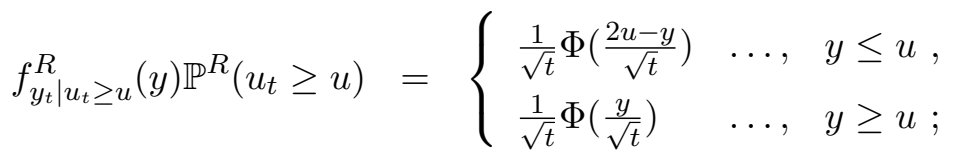

$$
\begin{aligned}
& \text { and } f_{y_{t}, u_{t}}^{R}(y, u)=\left\{\begin{array}{lll}
\frac{2(2 u-y)}{t} \frac{1}{\sqrt{t}} \Phi\left(\frac{2 u-y}{\sqrt{t}}\right) & \ldots, & y \leq u, \\
0 & \ldots, & y \geq u .
\end{array}\right.
\end{aligned}
$$

Then using the Radon-Nikodym derivative defined in (45) and doing a change of measure to Q, we get:

$$
\begin{aligned}
& f_{y_{t} \mid u_{t} \geq u}^{Q}(y) \mathbb{P}^{Q}\left(u_{t} \geq u\right)=\left\{\begin{array}{lll}
\frac{1}{\sqrt{t}} \Phi\left(\frac{2 u-y}{\sqrt{t}}\right) \exp \left(\eta y-\frac{1}{2} \eta^{2} t\right) & \ldots, & y \leq u \\
\frac{1}{\sqrt{t}} \Phi\left(\frac{y}{\sqrt{t}}\right) \exp \left(\eta y-\frac{1}{2} \eta^{2} t\right) & \ldots, & y>u
\end{array}\right. \\
& \text { and } f_{y_{t}, u_{t}}^{Q}(y, u)=\left\{\begin{array}{lll}
\frac{2(2 u-y)}{t} \frac{1}{\sqrt{t}} \Phi\left(\frac{2 u-y}{\sqrt{t}}\right) \exp \left(\eta y-\frac{1}{2} \eta^{2} t\right) & \ldots, & y \leq u \\
0 & \ldots, & y>u .
\end{array}\right.
\end{aligned}
$$

Using (49), we directly get,

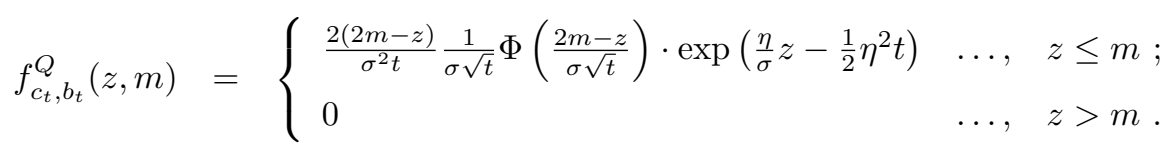

This is the relation in (27), noting $\nu=\sigma \eta$.

Also, using (48),

$$
\begin{aligned}
\mathbb{P}^{Q}\left(u_{t} \geq u\right)= & \int_{-\infty}^{u} \frac{1}{\sqrt{t}} \Phi\left(\frac{2 u-y}{\sqrt{t}}\right) \exp \left(\eta y-\frac{1}{2} \eta^{2} t\right) d y \\
& \quad+\int_{u}^{\infty} \frac{1}{\sqrt{t}} \Phi\left(\frac{y}{\sqrt{t}}\right) \exp \left(\eta y-\frac{1}{2} \eta^{2} t\right) d y \\
= & \exp (2 \eta u) \int_{-\infty}^{u} \frac{1}{\sqrt{t}} \Phi\left(\frac{y-2 u-\eta t}{\sqrt{t}}\right) d y+\int_{u}^{\infty} \frac{1}{\sqrt{t}} \Phi\left(\frac{y-\eta t}{\sqrt{t}}\right) d y \\
= & \exp (2 \eta u) \mathrm{N}\left(\frac{-u-\eta t}{\sqrt{t}}\right)+1-\mathrm{N}\left(\frac{u-\eta t}{\sqrt{t}}\right) . \\
\text { Hence, } f_{u_{t}}^{Q}(u)= & \frac{1}{\sqrt{t}} \Phi\left(\frac{u-\eta t}{\sqrt{t}}\right)+\exp (2 \eta u) \cdot \Phi\left(\frac{-u-\eta t}{\sqrt{t}}\right)-2 \eta \exp (2 \eta u) \mathrm{N}\left(\frac{-u-\eta t}{\sqrt{t}}\right) \\
= & 2 \cdot \frac{1}{\sqrt{t}} \Phi\left(\frac{u-\eta t}{\sqrt{t}}\right)-2 \eta \exp (2 \eta u) \cdot \mathrm{N}\left(\frac{-u-\eta t}{\sqrt{t}}\right) .
\end{aligned}
$$


Hence,

$$
f_{b_{t}}^{Q}(m)=2 \cdot \frac{1}{\sigma \sqrt{t}} \Phi\left(\frac{m-\sigma \eta t}{\sigma \sqrt{t}}\right)-\frac{2 \eta}{\sigma} \exp \left(\frac{2 \eta m}{\sigma}\right) \mathrm{N}\left(\frac{-m-\sigma \eta t}{\sigma \sqrt{t}}\right) .
$$

This is the same as in (29), as $\nu=\eta \sigma$.

\section{Appendix C}

\section{Computational Methods for Alternate Models}

In this Appendix, we provide a brief description of the computational methods used to value GWB for life under the different asset return models specified in Section 5.

\section{C.1 BSM Model}

For numerical computations, for each $n$, we evaluate the values of $l_{n}(x)$ and $g_{n}(x)$ as defined in (33) and (34) respectively, at $M+1$ evenly spaced points in the interval [0,1] with $M=$ 300. We then evaluate the integrals in (35) and (36) using simple linear interpolation. Because asset returns have lognormal distributions, these integrals can be evaluated easily. We describe in detail the procedure for evaluating the function $l_{n}(\cdot)$. An almost identical procedure with appropriate modifications based on (36) is used for evaluating $g_{n}(\cdot)$.

Let $x_{i}, 0 \leq i \leq M$ denote the $M+1$ points on the grid, with $x_{0}=0$ and $x_{M}=1$. For

$0 \leq i \leq M+1$, let $l_{i, n} \triangleq l_{n}\left(x_{i}\right)$ denote the values that the function $l_{n}(\cdot)$ takes on the grid-points. Suppose these values are available for some $n$. We first find the linear interpolation coefficients $A_{i, n}^{l}, B_{i, n}^{l} ; 0 \leq i \leq M$ as follows:

$$
\begin{aligned}
& A_{i, n}^{l}=\frac{l_{i+1, n}-l_{i, n}}{x_{i+1}-x_{i}} ; \\
& B_{i, n}^{l}=l_{i, n} .
\end{aligned}
$$

We use the following linear approximation for evaluating $l_{n}(x)$ at an arbitrary point $x$ :

$$
l_{n}(x) \approx A_{j, n}^{l}\left(x-x_{j}\right)+B_{j, n}^{l},
$$

where $j$ is such that $x_{j} \leq x<x_{j+1}$. 
Using the approximation in (51) in (35), we get

$$
\begin{aligned}
l_{n-1}(x)=e^{-\left(\lambda_{n}^{A}+r\right)} \cdot\{x & \cdot \int_{-\infty}^{\ln \left(\frac{q_{n}}{x}\right)}\left(\frac{q_{n}}{x}-e^{z}\right) \cdot \Phi(z ; \mu, \sigma) d z \\
& +\int_{\ln \left(\frac{q_{n}}{x}\right)}^{\ln \left(\frac{1+q_{n}}{x}\right)} l_{n}\left(x e^{z}-q_{n}\right) \cdot \Phi(z ; \mu, \sigma) d z \\
& \left.+x \cdot l_{n}(1) \cdot \int_{\ln \left(\frac{1+q_{n}}{x}\right)}^{\infty}\left(e^{z}-\frac{q_{n}}{x}\right) \cdot \Phi(z ; \mu, \sigma) d z\right\} \\
\approx e^{-\left(\lambda_{n}^{A}+r\right)} \cdot\left\{\int_{-\infty}^{\ln \left(\frac{q_{n}}{x}\right)}\left(q_{n}-x e^{z}\right) \cdot \Phi(z ; \mu, \sigma) d z\right. & \\
& +\sum_{i=1}^{M} \int_{\ln \left(\frac{x_{i-1}+q_{n}}{x}\right)}^{\ln \left(\frac{x_{i}+q_{n}}{x}\right)}\left(A_{i-1}^{l}\left(x e^{z}-q_{n}\right)+B_{i-1}^{l}\right) \cdot \Phi(z ; \mu, \sigma) d z \\
& \left.+l_{n}(1) \cdot \int_{\ln \left(\frac{1+q_{n}}{x}\right)}^{\infty}\left(x e^{z}-q_{n}\right) \cdot \Phi(z ; \mu, \sigma) d z\right\} .
\end{aligned}
$$

Now,

$$
\begin{aligned}
\int_{-\infty}^{y} \Phi(z ; \mu, \sigma) d z & =\mathrm{N}\left(\frac{y-\mu}{\sigma}\right) \\
& =\mathrm{N}\left(\frac{y}{\sigma}-\frac{r}{\sigma}+\frac{\sigma}{2}\right) \text { and } \\
\int_{y}^{\infty} \exp (z) \Phi(z ; \mu, \sigma) d z & =\exp \left(\mu+\frac{1}{2} \sigma^{2}\right) \mathrm{N}\left(\frac{-y}{\sigma}+\frac{r}{\sigma}+\frac{\sigma}{2}\right) \\
& =e^{r} \cdot \mathrm{N}\left(\frac{-y}{\sigma}+\frac{r}{\sigma}+\frac{\sigma}{2}\right) .
\end{aligned}
$$

Then, from (52),

$$
\begin{aligned}
l_{n-1}(x) \approx e^{-\lambda_{n}^{A}} & \cdot\left\{q_{n} e^{-r} \cdot \mathrm{N}\left(\frac{-\ln \frac{x}{q_{n}}}{\sigma}-\frac{r}{\sigma}+\frac{\sigma}{2}\right)-x \cdot \mathrm{N}\left(\frac{-\ln \frac{x}{q_{n}}}{\sigma}-\frac{r}{\sigma}-\frac{\sigma}{2}\right)\right. \\
& +x \cdot \sum_{i=1}^{M} A_{i-1}^{l} \cdot\left(\mathrm{N}\left(\frac{\ln \frac{x}{x_{i-1}+q_{n}}}{\sigma}+\frac{r}{\sigma}+\frac{\sigma}{2}\right)-\mathrm{N}\left(\frac{\ln \frac{x}{x_{i}+q_{n}}}{\sigma}+\frac{r}{\sigma}+\frac{\sigma}{2}\right)\right) \\
& +e^{-r} \sum_{i=1}^{M}\left(B_{i-1}^{l}-A_{i-1}^{l} q_{n}\right) \cdot\left(\mathrm{N}\left(\frac{\ln \frac{x}{x_{i-1}+q_{n}}}{\sigma}+\frac{r}{\sigma}-\frac{\sigma}{2}\right)-\mathrm{N}\left(\frac{\ln \frac{x}{x_{i}+q_{n}}}{\sigma}+\frac{r}{\sigma}-\frac{\sigma}{2}\right)\right) \\
& \left.+l_{M+1, n} \cdot\left(x \cdot \mathrm{N}\left(\frac{\ln \frac{x}{1+q_{n}}}{\sigma}+\frac{r}{\sigma}+\frac{\sigma}{2}\right)-q_{n} e^{-r} \cdot \mathrm{N}\left(\frac{\ln \frac{x}{1+q_{n}}}{\sigma}+\frac{r}{\sigma}-\frac{\sigma}{2}\right)\right)\right\} .
\end{aligned}
$$

The values of $A_{n}^{l}$ and $B_{n}^{l}$ for $n=\bar{N}$ should be set to 0 . Thus (53) can be used to successively evaluate $l_{n}(x)$ by backward substitution. Also, the values of $\mathrm{x}$ at which (53) is evaluated are fixed as the points $x_{i}, 0 \leq i \leq M$ on the grid. This allows us some further computational speed-ups as many quantities in (53) can be computed only once and then cached. 
Note that because moments of the normal distribution are available in a closed form, in general, we can use any polynomial interpolation method for approximating $l_{n}(\cdot)$ and $g_{n}(\cdot)$ and still avoid evaluating any integrals numerically.

\section{C.2 SILN Model}

For the SILN model, we use the product dynamics as given by (1) and Monte Carlo simulations (with 10000 sample paths) for valuing GWB. We simulate only the randomness due to the market risk factors. Mortality factors are accounted for directly in the spirit of (32).

For simulations, we need to generate the risk-free rate $r_{n}^{f}$ and the excess return of the VA fund over $r_{n}^{f}$ for each year. The excess return being log-normal and independent of $r_{n}^{f}$ in the SILN model can be generated in a straightforward way.

The generation of risk-free rate samples, from the two factor Gaussian process is more involved. From the specification of the short-rate model as given in (37), it is clear that at any time $t$, we have two state variables, $x_{1, t}$ and $x_{2, t}$. Under the Gaussian model, as we shall show shortly, the state variables $x_{1, T}, x_{2, T}$ and the factor $y_{T} \triangleq \int_{0}^{T} r_{s} d s$, which corresponds to the effective interest rate for the period $(0, T]$ are jointly normal. This then allows us to discretely sample the interest rate process and use a time step as large as the epoch interval in our simulations and speeds up the computations considerably.

The stochastic differential equations in (37) have the solution (see Oksendal [26]) :

$$
\begin{aligned}
& x_{1, t}=x_{1,0} \exp \left(-\kappa_{1} t\right)+\sigma_{1} \int_{0}^{t} \exp \left(-\kappa_{1}(t-s)\right) d Z_{s}^{1} \\
& x_{2, t}=x_{2,0} \exp \left(-\kappa_{2} t\right)+\sigma_{2} \int_{0}^{t} \exp \left(-\kappa_{2}(t-s)\right) d Z_{s}^{2} .
\end{aligned}
$$

It then follows that,

$$
\begin{aligned}
y_{T}=\int_{0}^{T} r_{t} d t= & \int_{0^{T}} x_{1, t} d t+\int_{0^{T}} x_{2, t} d t+\int_{0}^{T} b(t) d t \\
= & x_{1,0} \int_{0}^{T} \exp \left(-\kappa_{1} t\right) d t+\sigma_{1} \int_{0}^{T} \int_{0}^{t} \exp \left(-\kappa_{1}(t-s)\right) d Z_{s}^{1} d t \\
& +x_{2,0} \int_{0}^{T} \exp \left(-\kappa_{2} t\right) d t+\sigma_{2} \int_{0}^{T} \int_{0}^{t} \exp \left(-\kappa_{2}(t-s)\right) d Z_{s}^{2} d t+B(T) \\
= & x_{1,0} f\left(\kappa_{1}, T\right)+\sigma_{1} \int_{0}^{T} f\left(\kappa_{1}, T-s\right) d Z_{s}^{1} \\
& +x_{2,0} f\left(\kappa_{2}, T\right)+\sigma_{2} \int_{0}^{T} f\left(\kappa_{2}, T-s\right) d Z_{s}^{2}+B(T)
\end{aligned}
$$


where,

$$
\begin{aligned}
B(T) & =\int_{0}^{T} b(t) d t ; \\
f(\kappa, t) & \triangleq \frac{1-\exp (-\kappa t)}{\kappa} .
\end{aligned}
$$

From (54) and (55), it follows that $x_{1, T}, x_{2, T}$ and $y_{T}$ should be jointly normal. Further, using Ito isometry,

$$
\begin{aligned}
\operatorname{var}\left(x_{1, T}\right)= & \sigma_{1}^{2} \int_{0}^{t} \exp \left(-2 \kappa_{1}(t-s)\right) d s \\
= & \sigma_{1}^{2} f\left(2 \kappa_{1}, T\right) \\
\operatorname{var}\left(x_{1, T}\right)= & \sigma_{1}^{2} f\left(2 \kappa_{2}, T\right) \\
\operatorname{var}\left(y_{T}\right)= & \sigma_{1}^{2} \int_{0}^{T}\left(f\left(\kappa_{1}, T-s\right)\right)^{2} d s+\sigma_{2}^{2} \int_{0}^{T}\left(f\left(\kappa_{2}, T-s\right)\right)^{2} d s \\
& +2 \sigma_{1} \sigma_{2} \rho \int_{0}^{T} f\left(\kappa_{1}, T-s\right) \cdot f\left(\kappa_{2}, T-s\right) d s \\
= & \frac{\sigma_{1}^{2}}{\kappa_{1}^{2}}\left(T-2 f\left(\kappa_{1}, T\right)+f\left(2 \kappa_{1}, T\right)\right)+\frac{\sigma_{2}^{2}}{\kappa_{2}^{2}}\left(T-2 f\left(\kappa_{2}, T\right)+f\left(2 \kappa_{2}, T\right)\right) \\
& +2 \frac{\sigma_{1} \sigma_{2} \rho}{\kappa_{1} \kappa_{2}}\left(T-f\left(\kappa_{1}, T\right)-f\left(\kappa_{2}, T\right)+f\left(\kappa_{1}+\kappa_{2}, T\right)\right) .
\end{aligned}
$$

We can similarly find the covariances between the state variables and the effective interest rate:

$$
\begin{aligned}
\operatorname{cov}\left(x_{1, T}, x_{2, T}\right) & =\sigma_{1} \sigma_{2} \rho f\left(\kappa_{1}+\kappa_{2}, T\right) ; \\
\operatorname{cov}\left(x_{1, T}, y_{T}\right) & =\frac{\sigma_{1}^{2}}{\kappa_{1}}\left(f\left(\kappa_{1}, T\right)-f\left(2 \kappa_{1}, T\right)\right)+\frac{\sigma_{1} \sigma_{2} \rho}{\kappa_{2}}\left(f\left(\kappa_{1}, T\right)-f\left(\kappa_{1}+\kappa_{2}, T\right)\right) ; \\
\operatorname{cov}\left(x_{2, T}, y_{T}\right) & =\frac{\sigma_{1} \sigma_{2} \rho}{\kappa_{1}}\left(f\left(\kappa_{2}, T\right)-f\left(\kappa_{1}+\kappa_{2}, T\right)\right)+\frac{\sigma_{2}^{2}}{\kappa_{2}}\left(f\left(\kappa_{2}, T\right)-f\left(2 \kappa_{2}, T\right)\right) .
\end{aligned}
$$

The expressions in (56) and (57) can be used to directly generate a sample of the state variable $\left(x_{1, n+1}, x_{2, n+1}\right)$ and the one year risk-free rate $r_{n+1}^{f}$ from $\left(x_{1, n}, x_{2, n}\right)$.

Finally, the function $b(t)$ is adjusted so that the forward rates implied by the short rate model match the market forward rates. If $\gamma_{0}(t)$ denotes the time $t$ market forward rate, we must have

$$
\exp \left(-\int_{0}^{t} \gamma_{0}(s) d s\right)=\mathbb{E}^{\mathbb{Q}}\left[\exp \left(-y_{t}\right)\right] .
$$

Using (55) and (56) and the fact that $y_{T}$ is Gaussian, we get

$$
\begin{aligned}
\exp \left(-\int_{0}^{t} \gamma_{0}(s) d s\right)= & \exp \left(-x_{1,0} f\left(\kappa_{1}, t\right)-x_{2,0} f\left(\kappa_{2}, t\right)-B(t)+\frac{1}{2} \operatorname{var}\left(y_{t}\right)\right) . \\
\text { Hence, } b(t)= & \gamma_{0}(t)+\frac{d}{d t}\left(-x_{1,0} f\left(\kappa_{1}, t\right)-x_{2,0} f\left(\kappa_{2}, T\right)+\frac{1}{2} \operatorname{var}\left(y_{t}\right)\right) \\
= & \gamma_{0}(t)-x_{1,0} \exp \left(-\kappa_{1} t\right)-x_{2,0} \exp \left(-\kappa_{2} t\right) \\
& +\frac{1}{2}\left(\sigma_{1}^{2} \cdot\left(f\left(\kappa_{1}, t\right)\right)^{2}+\sigma_{2}^{2} \cdot\left(f\left(\kappa_{2}, t\right)\right)^{2}+2 \sigma_{1} \sigma_{2} \rho \cdot f\left(\kappa_{1}, t\right) f\left(\kappa_{2}, t\right)\right) .
\end{aligned}
$$




\section{C.3 SISV Model}

Unlike the BSM and SILN models, the SISV model does not offer many computational advantages. Like the SILN model, we use Monte Carlo simulations, again with 10,000 sample paths, to value GWB liabilities and revenue streams. We again simulate only the randomness coming from market factors, as the mortality related randomness can be directly incorporated in the pricing formulae. We discretely sample the interest rate process using the procedure described in Section C.2 for the SILN model.

To generate the excess VA fund returns, we first generate excess equity returns for one year using a discretized version of (39) with a small time step $\Delta=\frac{1}{250}$ years. This also gives us a sample of the other state variable in the system, i.e., $V_{n}$, or the instantaneous variance at the $n^{\text {th }}$ anniversary. The one year excess alternative investments return is obtained by directly sampling from a log-normal distribution with volatility $\sigma_{b}$. The one year excess portfolio return, i.e., $\ln \left(R_{n}^{s}\right)-r_{n}^{f}$ is then obtained by taking a weighted combination of the excess equity and the excess alterative investments' returns, with the weights selected in accordance with the composition chosen by the investor.

\section{REFERENCES}

[1] http://www.econ.yale.edu/ shiller/data.htm.

[2] M. Abramowitz and I. A. E. Stegun, Handbook of Mathematical Functions with Formulas, Graphs, and Mathematical Tables, 9th ed. New York: Dover, 1972.

[3] G. Bakshi, C. Cao, and Z. Chen, "Empirical Performance of Alternative Option Pricing Models," The Journal of Finance, vol. 52, no. 5, pp. 2003-2049, December 1997.

[4] L. Ballotta, G. Esposito, and S. Haberman, "Modelling the Fair Value of Annuities Contracts: The Impact of Interest Rate Risk and Mortality Risk ," December 2006, available at SSRN: http://ssrn.com/abstract=992211.

[5] L. Ballotta and S. Haberman, "Valuation of guaranteed annuity conversion options," Insurance: Mathematics and Economics, vol. 33, no. 1, pp. 87-108, August 2003.

[6] E. Biffis, "Affine processes for dynamic mortality and actuarial valuations," Insurance: Mathematics and Economics, vol. 37, no. 3, pp. 443-468, December 2005.

[7] E. Biffis and P. Millossovich, “The fair value of Guaranteed Annuity Options," Scandinavian Actuarial Journal, vol. 1, pp. 23-41, 2006.

[8] F. Black and M. Scholes, "The Pricing of Options and Corporate Liabilities," Journal of Political Economy, vol. 81, no. 3, pp. 637-654, May-June 1973.

[9] Z. Bodie, "Common Stocks as a Hedge Against Inflation," The Journal of Finance, vol. 31, no. 2, pp. 459-470, May 1975.

[10] P. P. Boyle and M. Hardy, "Guaranteed Annuity Options," ASTIN Bulletin, vol. 33, no. 2, pp. 125-152, January 2003.

[11] P. Boyle and E. Schwartz, "Equilibrium prices of guarantees under equity-linked contracts," Journal of Risk and Insurance, vol. 44, no. 4, pp. 639-680, December 1977. 
[12] M. Brennan and E. Schwartz, "The pricing of equity-linked life insurance policies with an asset value guarantee," Journal of Financial Economics, vol. 3, no. 3, pp. 195-213, June 1976.

[13] A. Cairns, D. Blake, and K. Dowd, "A two-factor model for Stochastic Mortaity with Parameter Uncertainty: Theory and Calibration," The Journal of Risk and Insurance, vol. 73, no. 4, pp. 687-718, 2006.

[14] Z. Chen, K. Vetzal, and P. A. Forsyth, “The Effect of Modelling Parameters on the Value of GMWB Guarantees," Insurance: Mathematics and Economics, vol. 43, no. 1, pp. 165-173, August 2008.

[15] C. C. Chu and Y. K. Kwok, "Valuation of guaranteed annuity options in affine term structure models," January 2005, available at SSRN: http://ssrn.com/abstract $=644224$.

[16] J. C. Cox, J. E. Ingersoll, and S. A. Ross, “A Theory of the Term Structure of Interest Rates," Econometrica, vol. 53, no. 2, pp. 385-407, March 1985.

[17] M. Dai, Y. K. Kwok, and J. Zong, “Guaranteed Minimum Withdrawal Benefit in Variable Annuities,” Mathematical Finance, vol. 18, no. 4, pp. 595-611, September 2008, available at SSRN: http://ssrn.com/abstract=964083.

[18] D. Duffie, Dynamic Asset pricing Theory, 3rd ed. Princeton University Press, 2001.

[19] S. Heston, "A closed-form solution for options with stochastic volatility with applications to bond and currency options," Review of Financial Studies, vol. 6, no. 2, pp. 327-343, Summer 1993.

[20] D. Holz, A. Kling, and J. Rub, “GMWB For Life: An analysis of Lifelong Withdrawal Guarantees," August 2007, presented at American Risk and Insurance Association 2007 Annual Meeting. Available at http://www.aria.org/meetings/2007papers/IE

[21] I. Karatzas and S. Shreve, Brownian Motion and Stochastic Calculus, $2^{\text {nd }}$ ed. Springer, 1991.

[22] J. H. Lee and D. R. Stock, "Embedded options and interest rate risk for insurance companies, banks and other financial institutions," The Quarterly Review of Economics and Finance, vol. 40, no. 2, pp. 169-187, Summer 2000.

[23] Mathworld, http://mathworld.wolfram.com/WhittakerDifferentialEquation.html, available at mathworld.wolfram.com. [Online]. Available: http://mathworld.wolfram.com/WhittakerDifferentialEquation.html

[24] M. A. Milevsky, S. D. Promislow, and V. R. Young, “Killing the Law of Large Numbers: Mortality Risk Premiums and the Sharpe Ratio," The Journal of Risk and Insurance, vol. 73, no. 4, pp. 673-686, 2006.

[25] M. A. Milevsky and T. S. Salisbury, "Financial valuation of guaranteed minimum withdrawal benefits," Insurance: Mathematics and Economics, vol. 38, no. 1, pp. 21-38, 2006.

[26] B. K. Oksendal, Stochastic Differential Equations: An Introduction with Applications, 5th ed. Springer, 1998.

[27] A. Pelsser, "Pricing and hedging guaranteed annuity options via static option replication," Insurance: Mathematics and Economics, vol. 33, no. 2, pp. 283-296, 2003.

[28] J. W. Snow, “One Thing We Can All Agree On,” The Wall Street Journal, January 20, 2005.

[29] O. Vasicek, "An equilibrium characterization of the term structure," Journal of Financial Economics, vol. 5, no. 2, pp. 177-188, November 1977.

[30] N. Wang, R. Gerrard, and S. Haberman, "The premium and the risk of a life policy in the presence of interest rate fluctuations," Insurance: Mathematics and Economics, vol. 35, no. 3, pp. 537-551, December 2004. 